\title{
FROM APOLLONIUS TO ZAREMBA: LOCAL-GLOBAL PHENOMENA IN THIN ORBITS
}

\author{
ALEX KONTOROVICH
}

\begin{abstract}
We discuss a number of natural problems in arithmetic, arising in completely unrelated settings, which turn out to have a common formulation involving "thin" orbits. These include the local-global problem for integral Apollonian gaskets and Zaremba's Conjecture on finite continued fractions with absolutely bounded partial quotients. Though these problems could have been posed by the ancient Greeks, recent progress comes from a pleasant synthesis of modern techniques from a variety of fields, including harmonic analysis, algebra, geometry, combinatorics, and dynamics. We describe the problems, partial progress, and some of the tools alluded to above.
\end{abstract}

\section{Contents}

1. Introduction

2. Zaremba's Conjecture

3. Integral Apollonian gaskets

4. The thin Pythagorean problem

5. The circle method: tools and proofs $\quad 217$

Acknowledgments

About the author

References

\section{INTRODUCTION}

In this article we will discuss recent developments on several seemingly unrelated arithmetic problems, which each boil down to the same issue of proving a "localglobal principle for thin orbits". In each of these problems, we study the orbit

$$
\mathcal{O}=\Gamma \cdot \mathbf{v}_{0},
$$

of some given vector $\mathbf{v}_{0} \in \mathbb{Z}^{d}$, under the action of some given group or semigroup, $\Gamma$, (under multiplication) of $d$-by- $d$ integer matrices. It will turn out that the orbits arising naturally in our problems are thin; roughly speaking, this means that each orbit is degenerate in its algebro-geometric closure, containing relatively very few points.

Received by the editors August 15, 2012, and, in revised form, November 4, 2012.

2010 Mathematics Subject Classification. Primary 11F41, 11J70, 11P55, 20H10, 22E40.

Partially supported by NSF grants DMS-1209373, DMS-1064214 and DMS-1001252. 
Each of the problems then takes another vector $\mathbf{w}_{0} \in \mathbb{Z}^{d}$, and for the standard inner product $\langle\cdot, \cdot\rangle$ on $\mathbb{R}^{d}$, forms the set

$$
\mathscr{S}:=\left\langle\mathbf{w}_{0}, \mathcal{O}\right\rangle \subset \mathbb{Z}
$$

of integers, asking what numbers are in $\mathscr{S}$.

For an integer $q \geq 1$, the projection map

$$
\mathbb{Z} \rightarrow \mathbb{Z} / q \mathbb{Z}
$$

can give an obvious obstruction to membership. Let $\mathscr{S}(\bmod q)$ be the image of this projection,

$$
\mathscr{S}(\bmod q):=\{s(\bmod q): s \in \mathscr{S}\} \subset \mathbb{Z} / q \mathbb{Z} .
$$

For example, suppose that any number in $\mathscr{S}$ leaves a remainder of 1,2 or 3 when divided by 4 , that is, $\mathscr{S}(\bmod 4)=\{1,2,3\}$. Then one can conclude, without any further consideration, that $10^{10^{10}} \notin \mathscr{S}$, since $10^{10^{10}} \equiv 0(\bmod 4)$. This is called a local obstruction. Call $n$ admissible if it avoids all local obstructions,

$$
n \in \mathscr{S}(\bmod q), \quad \text { for all } q \geq 1 \text {. }
$$

In many applications, the set $\mathscr{S}(\bmod q)$ is significantly easier to analyze than the set $\mathscr{S}$ itself. But a local to global phenomenon predicts that, if $n$ is admissible, then in fact $n \in \mathscr{S}$, thereby reducing the seemingly more difficult problem to the easier one.

It is the combination of these concepts, $(i)$ thin orbits, and (ii) local-global phenomena, which will turn out to be the "beef" of the problems we intend to discuss.

1.1. Outline. We begin in $\$ 2$ with Zaremba's Conjecture. We will explain how this problem arose naturally in the study of "good lattice points" for quasi-Monte Carlo methods in multi-dimensional numerical integration, and how it also has applications to the linear congruential method for pseudo-random number generators. But the assertion of the conjecture is a statement about continued fraction expansions of rational numbers, and as such is so elementary that Euclid himself could have posed it. We will discuss recent progress by Bourgain and the author, proving a density version of the conjecture.

We change our focus in $\S 3$ to the ancient geometer Apollonius of Perga. As we will explain, his straight-edge and compass construction of tangent circles, when iterated ad infinitum, gives rise to a beautiful fractal circle packing in the plane, such as that shown in Figure 1. Recall that the curvature of a circle is just one over its radius. For special configurations, all the curvatures of circles in the given packing turn out to be integers; these are the numbers shown in Figure 11. In $\$ 3$ we will present progress on the problem, which integers appear? It was recently proved by Bourgain and the author that almost every admissible number appears.

In \$4, also stemming from Greek mathematics, we describe a local-global problem for a thin orbit of Pythagorean triples, as will be defined there. This problem is a variant of the so-called Affine Sieve, recently introduced by Bourgain, Gamburd, and Sarnak. We will explain an "almost" local-global theorem in this context due to Bourgain and the author.

Finally, these three problems are reformulated to the aforementioned common umbrella in \$5, where some of the ingredients of the proofs are sketched. The problems do not naturally fit in an established area of research, having no $L$-functions 


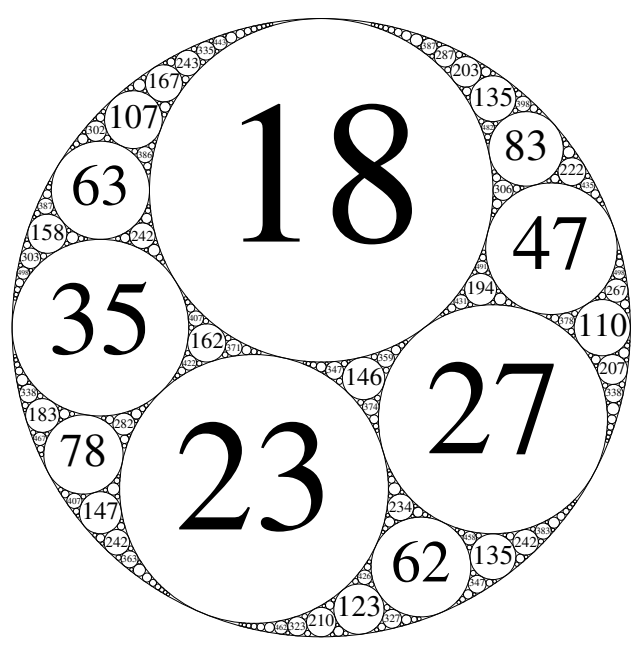

Figure 1. An integral Apollonian gasket.

or Hecke theory (though they are unquestionably problems about whole numbers), not being part of the Langlands Program (though involving automorphic forms and representations), nor falling under the purview of the classical circle method or sieve, which attempt to solve equations or produce primes in polynomials (here it is not polynomials that generate points, but the aforementioned matrix actions). Instead the proofs borrow bits and pieces from these fields and others, the major tools including analysis (the circle method, exponential sum bounds, infinite volume spectral theory), algebra (strong approximation, Zariski density, spin and orthogonal groups associated to quadratic forms, representation theory), geometry (hyperbolic manifolds, circle packings, Diophantine approximation), combinatorics (sum-product, expander graphs, spectral gaps), and dynamics (ergodic theory, mixing rates, the thermodynamic formalism). We aim to highlight some of these ingredients throughout.

1.2. Notation. We use the following standard notation. A quantity is defined via the symbol ":=", and a concept being defined is italicized. Write $f \sim g$ for $f / g \rightarrow 1$, $f=o(g)$ for $f / g \rightarrow 0$, and $f=O(g)$ or $f \ll g$ for $f \leq C g$. Here $C>0$ is called an implied constant, and is absolute unless otherwise specified. Moreover, $f \asymp g$ means $f \ll g \ll f$. We use $e(x)=e^{2 \pi i x}$. The cardinality of a finite set $S$ is written as $|S|$ or $\# S$. The transpose of a vector $\mathbf{v}$ is written $\mathbf{v}^{t}$. The meaning of algebraic symbols can change from section to section; for example the (semi)group $\Gamma$ and quadratic form $Q$ will vary depending on the context.

\section{Zaremba's ConjeCture}

Countless applications require pseudo-random numbers: deterministic algorithms which "behave randomly". Probably the simplest, oldest, and best known among these is the so-called linear congruential method: For some starting seed $x_{0}$, iterate the map

$$
x \mapsto b x+c \quad(\bmod d) .
$$




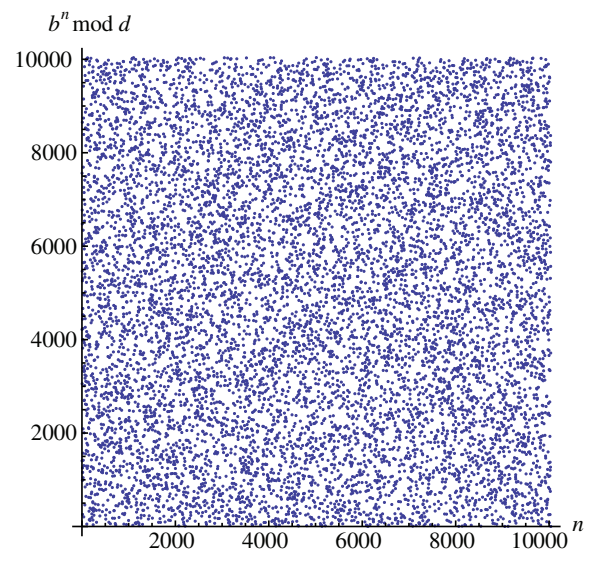

(a) Multiplier $b=4217$.

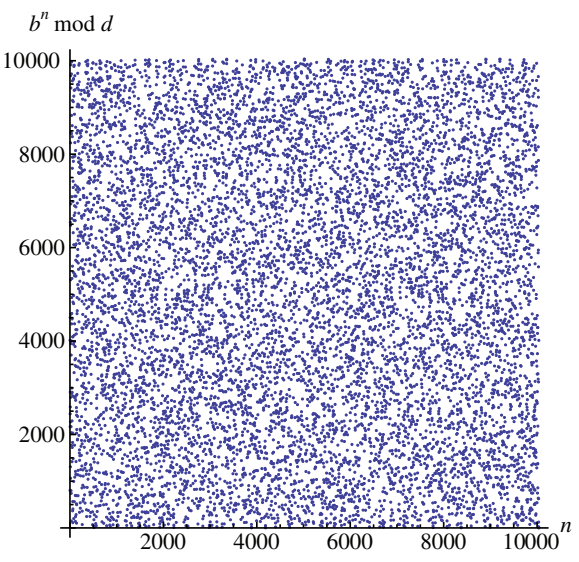

(b) Multiplier $b=4015$.

FiguRE 2. Graphs of the map (2.2) with prime modulus $d=10037$ and multiplier $b$ as shown.

Here $b$ is called the multiplier, $c$ the shift, and $d$ the modulus. For simplicity, we consider the homogeneous case $c=0$. To have as long a sequence as possible, take $d$ to be prime, and $b$ a primitive root $\bmod d$, that is, a generator of the cyclic group $(\mathbb{Z} / d \mathbb{Z})^{\times}$. In this case we may as well start with the seed $x_{0}=1$; then the iterates of (2.1) are nothing more than the map

$$
n \mapsto b^{n} \quad(\bmod d) .
$$

We show graphs of this map in Figure 2 for the prime $d=10037$, with two choices of roots, $b=4217$ and $b=4015$. In both cases, the graphs "look" random, in that, given $b$ and $n$, it is hard to guess where $b^{n}(\bmod d)$ will lie (without just computing). Similarly, given $b$ and $b^{n}(\bmod d)$, it is typically difficult to determine $n$; this is the classical problem of computing a discrete logarithm.

A slightly more rigorous statistical test for randomness is the serial correlation of pairs: How well can we guess where $b^{n+1}$ is, knowing $b^{n}$ ? To this end, we plot in Figure 3 these pairs, or what is the same, the pairs

$$
\left\{\left(\frac{b^{n}}{d}, \frac{b^{n+1}}{d}\right)(\bmod 1)\right\}_{n=1}^{d} \subset \mathbb{R}^{2} / \mathbb{Z}^{2}
$$

in the unit square, with the previous choices of modulus and multiplier. Focus first on Figure 3a: it looks like a fantastically equidistributed grid. Keep in mind that the mesh in each coordinate is of size $1 / d \approx 1 / 10000$, so we have $(10000)^{2}$ points from which to choose, yet we are only plotting 10000 points, square-root the total number of options.

On the other hand, look at Figure 3b: these parameters make a terrible random number generator! Given the first few terms in this sequence $\left(x_{1}, x_{2}, x_{3}, \ldots, x_{k}\right)$, with $x_{n}=b^{n} / d(\bmod 1)$, we simply plot the pairs $\left(x_{1}, x_{2}\right),\left(x_{2}, x_{3}\right), \ldots,\left(x_{k-1}, x_{k}\right)$, and then have a $1: 5$ guess for where $x_{k+1}$ will be.

A related phenomenon also appears in two-dimensional numerical integration: Suppose that you wish to integrate a "nice" function $f$ on $\mathbb{R}^{2} / \mathbb{Z}^{2} \cong[0,1) \times[0,1)$, 


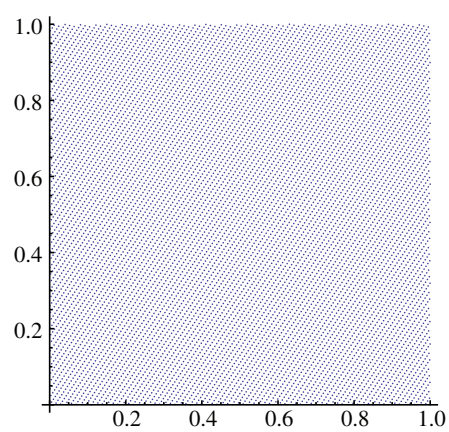

(a) Multiplier $b=4217$.

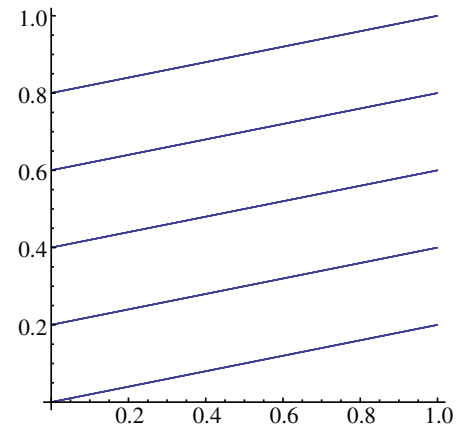

(b) Multiplier $b=4015$.

Figure 3. Plots of the points (2.3) for the same choices of modulus $d=10037$ and multipliers as in Figure 2.

say of finite variation, $V(f)<\infty$, where

$$
V(f):=\int_{0}^{1} \int_{0}^{1}\left(|f|+\left|\frac{\partial}{\partial x} f\right|+\left|\frac{\partial}{\partial y} f\right|+\left|\frac{\partial^{2}}{\partial x \partial y} f\right|\right) d x d y
$$

The idea is to take a large sample of points $\mathcal{Z}$ in $\mathbb{R}^{2} / \mathbb{Z}^{2}$ and approximate the integral by the average of $f(z), z \in \mathcal{Z}$. For this to be a good approximation one obviously needs that $f$ does not vary much in a small ball, and that the points of $\mathcal{Z}$ are well distributed throughout $\mathbb{R}^{2} / \mathbb{Z}^{2}$. In fact, the famous Koksma-Hlawka inequality (see [Nie78, p. 966]) states, rather beautifully, that this is all that one needs to take into account:

$$
\left|\int_{0}^{1} \int_{0}^{1} f(x, y) d x d y-\frac{1}{|\mathcal{Z}|} \sum_{z \in \mathcal{Z}} f(z)\right| \leq C \cdot V(f) \cdot \operatorname{Disc}(\mathcal{Z}) .
$$

Here $C>0$ is an absolute constant, and Disc is the discrepancy of the set $\mathcal{Z}$, defined as follows. Take a rectangle $R=[a, b] \times[c, d] \subset \mathbb{R}^{2} / \mathbb{Z}^{2}$. One would like the fraction of points in $R$ to be close to its area, so set

$$
\operatorname{Disc}(\mathcal{Z}):=\sup _{R \subset \mathbb{R}^{2} / \mathbb{Z}^{2}}\left|\frac{\#(\mathcal{Z} \cap R)}{\# \mathcal{Z}}-\operatorname{Area}(R)\right| .
$$

It is elementary that for a growing family $\mathcal{Z}^{(k)} \subset \mathbb{R}^{2} / \mathbb{Z}^{2},\left|\mathcal{Z}^{(k)}\right| \rightarrow \infty$, the discrepancy $\operatorname{Disc}\left(\mathcal{Z}^{(k)}\right)$ decays to 0 if and only if $\mathcal{Z}^{(k)}$ becomes equidistributed in $\mathbb{R}^{2} / \mathbb{Z}^{2}$. But more than just indicating equidistribution, the discrepancy measures the rate. For example, observe that for any finite sample set $\mathcal{Z}$, we have the lower bound $\operatorname{Disc}(\mathcal{Z}) \geq 1 /|\mathcal{Z}|$. Indeed, take a family of rectangles $R$ zooming in on a single point in $\mathcal{Z}$; the proportion of points in $R$ is always $1 /|\mathcal{Z}|$, while the area of $R$ can be made arbitrarily small. It turns out there is a sharpest possible lower bound, due to Schmidt Sch72:

$$
\text { for any finite } \mathcal{Z} \subset S, \quad \operatorname{Disc}(\mathcal{Z}) \gg \frac{\log |\mathcal{Z}|}{|\mathcal{Z}|} .
$$

Standard Monte Carlo integration is the process of computing the integral of $f$ by just sampling $z \in \mathcal{Z}$ according to the uniform measure; the Central Limit 
Theorem then predicts that

$$
\operatorname{Disc}(\mathcal{Z}) \approx \frac{1}{|\mathcal{Z}|^{1 / 2}}
$$

ignoring $\log \log$ factors. So comparing (2.5) to (2.4), it is clear that uniformly sampled sequences are far from optimal in numerical integration. Alternatively, one could take $\mathcal{Z}$ to be an evenly spaced $d$-by- $d$ grid,

$$
\mathcal{Z}=\{(i / d, j / d): 0 \leq i, j<d\},
$$

with $|\mathcal{Z}|=d^{2}$. But then the rectangle $[\varepsilon, 1 / d-\varepsilon] \times[0,1]$ contains no grid points while its area is almost $1 / d=1 /|\mathcal{Z}|^{1 / 2}$, again giving (2.5).

In the quasi-Monte Carlo method, rather than sampling uniformly, one tries to find a special sample set $\mathcal{Z}$ to come as close as possible to the optimal discrepancy (2.4). Ideally, such a set $\mathcal{Z}$ would also be quickly and easily constructible by a computer algorithm. Not surprisingly, the set $\mathcal{Z}$ illustrated in Figure 3 a makes an excellent sample set. It was this problem which led Zaremba to his theorem and conjecture, described below.

Returning to our initial discussion, observe that the sequence (2.3) is essentially (since $b$ is a generator) the same as

$$
\mathcal{Z}_{b, d}:=\left\{\left(\frac{n}{d}, \frac{b n}{d}\right)\right\}_{n=1}^{d}(\bmod 1) .
$$

And this is nothing more than a graph of our first map (2.1). Now it is clear that both Figures $3 \mathrm{a}$ and $3 \mathrm{~b}$ are "lines", but the first must be "close to a line with irrational slope", causing the equidistribution. This Diophantine property is best described in terms of continued fractions, as follows.

For $x \in(0,1)$, we use the notation

$$
x=\left[a_{1}, a_{2}, \ldots\right]
$$

for the continued fraction expansion

$$
x=\frac{1}{a_{1}+\frac{1}{a_{2}+\ddots} .}
$$

The integers $a_{j} \geq 1$ are called partial quotients of $x$. Rational numbers have finite continued fraction expansions.

One is then immediately prompted to study the continued fraction expansions of the "slopes" b/d in Figure 3

$$
\begin{aligned}
4217 / 10037 & =[2,2,1,1,1,2,2,2,1,2,2,1,2], \\
4015 / 10037 & =[2,2,2007] .
\end{aligned}
$$

Note the gigantic partial quotient 2007 in the second expression, while the partial quotients in the first are all 1's and 2's. Observations of this kind naturally led Zaremba to the following

Theorem 2.7 (Zaremba, 1966 [Zar66, Corollary 5.2]). Fix $(b, d)=1$ with $b / d=$ $\left[a_{1}, a_{2}, \ldots, a_{k}\right]$ and let $A:=\max a_{j}$. Then for $\mathcal{Z}_{b, d}$ given in (2.6),

$$
\operatorname{Disc}\left(\mathcal{Z}_{b, d}\right) \leq\left(\frac{4 A}{\log (A+1)}+\frac{4 A+1}{\log d}\right) \frac{\log d}{d}
$$


Since $\left|\mathcal{Z}_{b, d}\right|=d$, comparing the upper bound (2.8) to Schmidt's lower bound (2.4) shows that the sequences (2.6) are essentially best possible, up to the "constant" $A$ (and this optimal equidistribution is precisely what we observe visually in Figure 33). But the previous sentence is complete nonsense: $A$ is not constant at all; it depends on $d 11$ and Figure $3 \mathrm{~b}$ perfectly illustrates what can go wrong.

With this motivation, Zaremba predicted that in fact $A$ can be taken constant:

Conjecture Z (Zaremba, 1972 [Zar72, p. 76]). Every natural number is the denominator of a reduced fraction whose partial quotients are absolutely bounded.

That is, there exists some absolute $A>1$ so that for each $d \geq 1$, there is some $(b, d)=1$, so that $b / d=\left[a_{1}, \ldots, a_{k}\right]$ with $\max a_{j} \leq A$.

Zaremba even suggested a sufficient value for $A$, namely $A=5$. So this is really a problem that could have been posed in Book VII of the Elements (after Euclid's algorithm): Using the partial quotients $a_{j} \in\{1, \ldots, 5\}$, does the set of (reduced) fractions with expansion $\left[a_{1}, \ldots, a_{k}\right]$ contain every integer as a denominator? The reason for Zaremba's guess $A=5$ is simply that it is false for $A=4$, as we now explain. First some more notation.

Let $\mathscr{R}_{A}$ be the set of rationals with the desired property that all partial quotients are at most $A$,

$$
\mathscr{R}_{A}:=\left\{\frac{b}{d}=\left[a_{1}, \ldots, a_{k}\right]:(b, d)=1, \text { and } a_{j} \leq A, \forall j\right\} ;
$$

and let $\mathscr{D}_{A}$ be the set of denominators which arise,

$$
\mathscr{D}_{A}:=\left\{d: \exists(b, d)=1 \text { with } \frac{b}{d} \in \mathscr{R}_{A}\right\} .
$$

Then Zaremba's conjecture is that $\mathscr{D}_{5}=\mathbb{N}$, and we claim that this is false for $\mathscr{D}_{4}$. Indeed, $6 \notin \mathscr{D}_{4}$ : the only numerators to try are 1 and 5 , but the continued fraction expansion of $1 / 6$ is just [6], and $5 / 6=[1,5]$, so the largest partial quotient in both is too big.

That said, there are only two other numbers, 54 and 150, known to be missing from $\mathscr{D}_{4}$ (see [OEI]), leading one to ask what happens if a finite number of exceptions is permitted. Indeed, Niederreiter [Nie78, p. 990] conjectured in 1978 that for $A=3$, $\mathscr{D}_{3}$ already contains every sufficiently large number; we write this as

$$
\mathscr{D}_{3} \supset \mathbb{N}_{\gg 1} \text {. }
$$

With lots more computational capacity and evidence, Hensley almost 20 years later Hen96] conjectured even more boldly that the same holds already for $A=2$ :

$$
\mathscr{D}_{2} \supset \mathbb{N}_{\gg 1} \text {. }
$$

Lest the reader be tempted to one-up them all, let us consider the case $A=1$. Here $\mathscr{R}_{1}$ contains only continued fractions of the form $[1, \ldots, 1]$, and these are quotients of consecutive Fibonacci numbers $F_{n}$,

$$
\mathscr{R}_{1}=\left\{F_{n} / F_{n+1}\right\} .
$$

So $\mathscr{D}_{1}=\left\{F_{n}\right\}$ is just the Fibonacci numbers, and this is an exponentially thin sequence.

\footnotetext{
${ }^{1}$ The value $A$ also depends on $b$, but the important variable for applications is $\left|\mathcal{Z}_{b, d}\right|=d$.
} 
In fact, Hensley conjectured something much stronger than (2.9). First some more notation. Let $\mathscr{C}_{A}$ be the set of limit points of $\mathscr{R}_{A}$,

$$
\mathscr{C}_{A}:=\left\{\left[a_{1}, a_{2}, \ldots\right]: a_{j} \leq A, \forall j\right\} .
$$

To get our bearings, consider again the case $A=1$. Then $\mathscr{C}_{1}=\{1 / \varphi\}$ is just the singleton consisting of the reciprocal of the golden mean.

Now take $A=2$. Consider the unit interval $[0,1]$. The numbers in the range $(1 / 2,1]$ have first partial quotient $a_{1}=1$, and those in $(1 / 3,1 / 2]$ have first partial quotient $a_{1}=2$. The remaining interval $[0,1 / 3]$ has numbers whose first partial quotient is already too big, and thus is cut out. We repeat in this way, cutting out intervals for each partial quotient, and arriving at $\mathscr{C}_{2}$; see Figure 4

For any $A \geq 1$, the Cantor-like set $\mathscr{C}_{A}$ has some Hausdorff dimension

$$
\delta_{A}:=\operatorname{dim}\left(\mathscr{C}_{A}\right),
$$

which we recall is defined as the infimum of all $s \geq 0$ for which

$$
\inf _{\cup_{j} B_{j} \supset \mathscr{C}_{A}}\left\{\sum_{j} r\left(B_{j}\right)^{s}\right\}
$$

vanishes. The infimum in (2.11) is over collections $\left\{B_{j}\right\}_{j}$ of open balls (intervals) which cover $\mathscr{C}_{A}$, and $r\left(B_{j}\right)$ is the radius of $B_{j}$ (half the length of the interval).

Clearly $\delta_{1}=0$, since $\mathscr{C}_{1}$ is a single point. There is a substantial literature estimating the dimension $\delta_{2}$ which we will not survey, but the current record is due to Jenkinson and Pollicott [JP01], whose superexponential algorithm estimates

$$
\delta_{2}=0.5312805062772051416244686 \cdots .
$$

If we relax the bound $A$, the Cantor sets increase, as do their dimensions. In fact, Hensley Hen92 determined the asymptotic expansion, which to first order is

$$
\delta_{A}=1-\frac{6}{\pi^{2} A}+o\left(\frac{1}{A}\right),
$$

as $A \rightarrow \infty$. In particular, the dimension can be made arbitrarily close to 1 by taking $A$ large.

$$
\begin{aligned}
& \mathscr{C}_{2}^{(1)}=\left\{\left[a_{1}, \ldots\right]: a_{1} \leq 2\right\}: \\
& \mathscr{C}_{2}^{(2)}=\left\{\left[a_{1}, \ldots\right]: a_{1}, a_{2} \leq 2\right\}: \\
& \vdots \\
& \mathscr{C}_{2}^{(\infty)}=\mathscr{C}_{2}:
\end{aligned}
$$

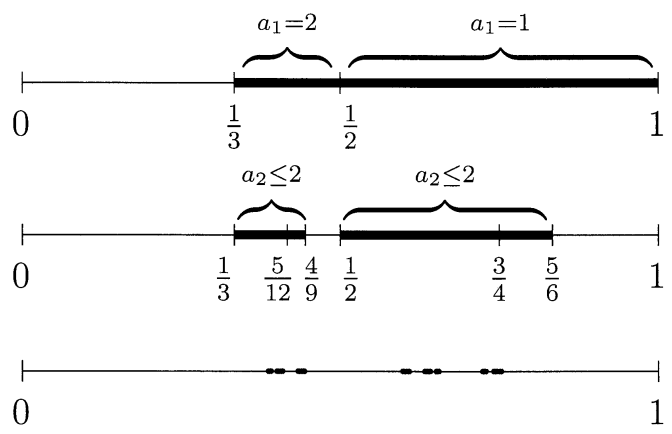

Figure 4. The Cantor set $\mathscr{C}_{2}=\bigcap_{k=1}^{\infty} \mathscr{C}_{2}^{(k)}$, where $\mathscr{C}_{2}^{(k)}=$ $\left\{\left[a_{1}, \ldots, a_{j}, \ldots, a_{k}, \ldots\right]: a_{j} \leq A\right.$ for all $\left.1 \leq j \leq k\right\}$ restricts only the first $k$ partial quotients. 
We can now explain Hensley's stronger conjecture. His observation is that one need not only consider restricting the partial quotients $a_{j}$ to the full interval $[1, A]$, one can allow more flexibility by fixing any finite "alphabet" $\mathcal{A} \subset \mathbb{N}$, and restricting the partial quotients to the "letters" in this alphabet. To this end, let $\mathscr{C}_{\mathcal{A}}$ be the Cantor set

$$
\mathscr{C}_{\mathcal{A}}:=\left\{\left[a_{1}, a_{2}, \ldots\right]: a_{j} \in \mathcal{A}, \forall j \geq 1\right\},
$$

and similarly let $\mathscr{R}_{\mathcal{A}}$ be the partial convergents to $\mathscr{C}_{\mathcal{A}}, \mathscr{D}_{\mathcal{A}}$ the denominators of $\mathscr{R}_{\mathcal{A}}$, and $\delta_{\mathcal{A}}$ the Hausdorff dimension of $\mathscr{C}_{\mathcal{A}}$. Then Hensley's elegant claim is the following

Conjecture 2.14 (Hensley, 1996 [Hen96, Conjecture 3, p. 16]).

$$
\mathscr{D}_{\mathcal{A}} \supset \mathbb{N}_{\gg 1} \quad \Longleftrightarrow \quad \delta_{\mathcal{A}}>1 / 2 \text {. }
$$

Observe in particular that $\delta_{2}$ in (2.12) exceeds $1 / 2$, and hence Hensley's full conjecture (2.15) implies the special case $A=2$ in (2.9).

Here is some heuristic evidence in favor of (2.15). Let us visualize the set $\mathscr{R}_{\mathcal{A}}$ of rationals, by grading each fraction according to the denominator. That is, plot each fraction $b / d$ at height $d$, showing the set

$$
\left\{\left(\frac{b}{d}, d\right): \frac{b}{d} \in \mathscr{R}_{\mathcal{A}}, \quad(b, d)=1\right\} .
$$

We show this plot in Figure 5 for $\mathcal{A}=\{1,2\}$ truncated at height $N=10000$, and in Figure 5b for $\mathcal{A}=\{1,2,3,4,5\}$ truncated at height $N=1000$. We give a name to this truncation, defining

$$
\mathscr{R}_{\mathcal{A}}(N):=\left\{\frac{b}{d} \in \mathscr{R}_{\mathcal{A}}:(b, d)=1,1 \leq b<d<N\right\} .
$$

Observe that the "vertical tentacles" in Figure 5 emanate from points on the $x$-axis lying in the Cantor sets $\mathscr{C}_{\mathcal{A}}$; compare Figures 5a and 4. Moreover, note that if at least one point has been placed at height $d$, then $d \in \mathscr{D}_{\mathcal{A}}$. That is, the "beef" of this problem boils down to, what are the projections of the plots in Figure 5 to the $y$-axis? In particular, does every (sufficiently large) integer appear?

The first question to address is, How big is $\left|\mathscr{R}_{\mathcal{A}}(N)\right|$, that is, how many points are being plotted in Figures $5 \mathrm{~b}$ and 5b? Hensley [Hen89] showed that, as $N \rightarrow \infty$,

$$
\# \mathscr{R}_{\mathcal{A}}(N) \asymp N^{2 \delta_{\mathcal{A}}},
$$

where the implied constant can depend on $\mathcal{A}$. (Hensley proved this for the alphabet $\mathcal{A}=\{1,2, \ldots, A\}$, but the same proof works for an arbitrary finite $\mathcal{A}$.)

Now, the $\Longrightarrow$ direction of (2.15) is trivial. Indeed, let

$$
\mathscr{D}_{\mathcal{A}}(N):=\mathscr{D}_{\mathcal{A}} \cap[1, N],
$$

so that the left-hand side of (2.15) is equivalent to

$$
\# \mathscr{D}_{\mathcal{A}}(N)=N+O(1), \quad \text { as } N \rightarrow \infty .
$$

Then it is clear that $\# \mathscr{R}_{\mathcal{A}}(N)$ counts $d$ 's with multiplicity, whereas $\# \mathscr{D}_{\mathcal{A}}(N)$ counts each appearing $d$ only once; hence

$$
\# \mathscr{D}_{\mathcal{A}}(N) \leq \# \mathscr{R}_{\mathcal{A}}(N) \stackrel{\sqrt{2.17}}{\ll} N^{2 \delta_{\mathcal{A}}} .
$$

So if (2.18) holds, then (2.19) implies that $2 \delta_{\mathcal{A}}$ must be at least 1 . 


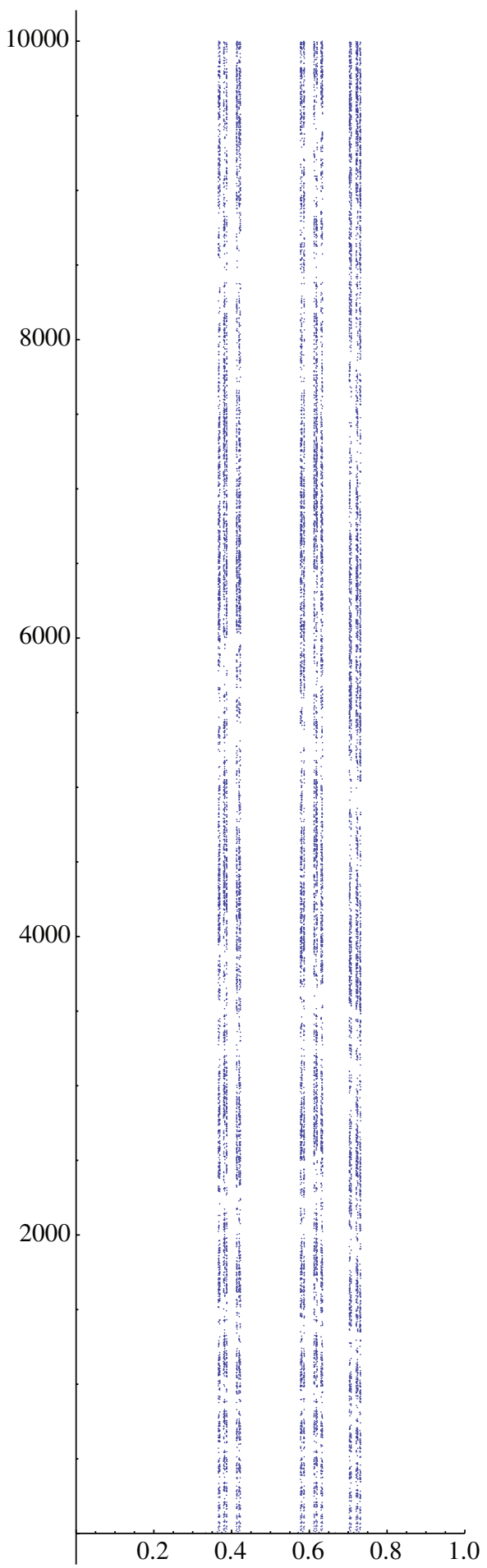

(a) $A=2, N=10000$.

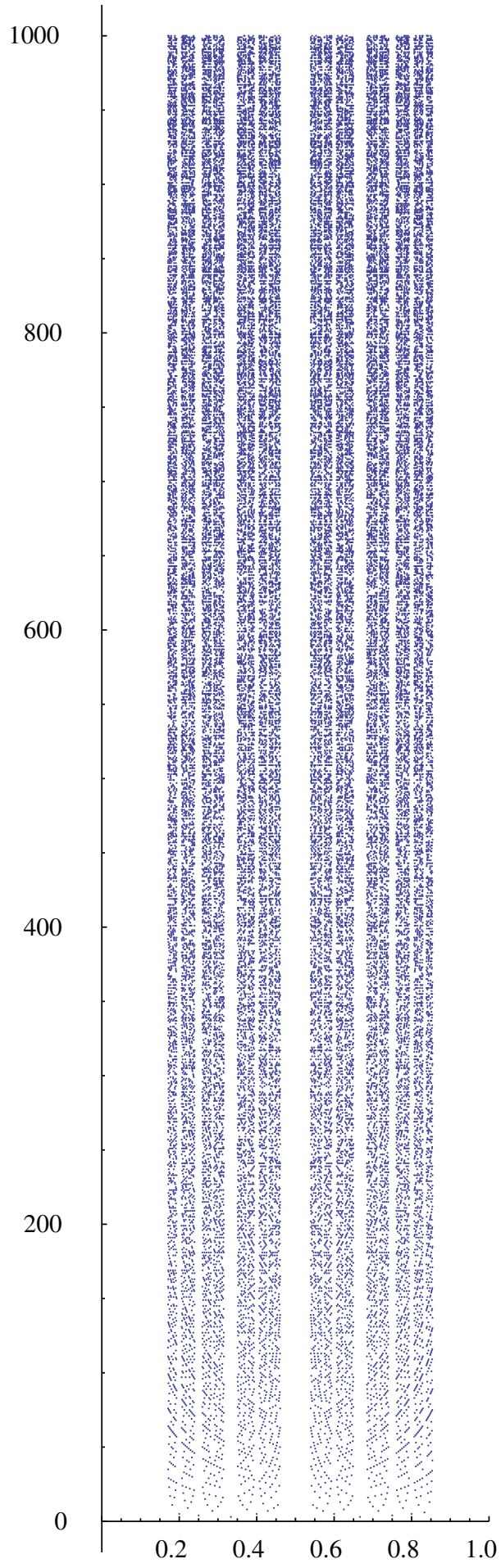

(b) $A=5, N=1000$.

Figure 5. For each $b / d \in \mathscr{R}_{A}(N)$, plot $b / d$ versus $d$, with $A$ and truncation parameter $N$ as shown. 
A caveat: we do not know how to verify (2.18) for a single alphabet! Nevertheless the content of Hensley's Conjecture is clearly the opposite $\Longleftarrow$ direction. Here is some evidence in favor of this claim.

An old theorem of Marstrand's Mar54 states the following. Let $E \subset[0,1] \times$ $[0,1]$ be a Hausdorff measurable set having Hausdorff dimension $\alpha>1$. Then the projection of $E$ into a line of slope $\tan \theta$ is "large," for Lebesgue-almost every $\theta \in \mathbb{R} / 2 \pi \mathbb{Z}$. Here "large" means of positive Lebesgue measure. One may thus heuristically think of (2.16) as $E$ above, with (2.17) suggesting the "dimension" $\alpha=2 \delta_{\mathcal{A}}$. Then $\mathscr{D}_{\mathcal{A}}$ is the projection of this $E$ to the $y$-axis, and it should be "large" according to the analogy. Marstrand's theorem says nothing about an individual line, and does not apply to the countable set (2.16), so the analogy cannot be furthered in any meaningful way. Nevertheless, we see the condition $\alpha>1$ is converted into $2 \delta_{\mathcal{A}}>1$, giving evidence for the $\Longleftarrow$ direction of (2.15).

For another heuristic, if one uniformly samples $N^{2 \delta}$ pairs $(b, d)$ out of the integers up to $N$, a given $d$ is expected to appear with multiplicity roughly $N^{2 \delta-1}$. For $\delta>1 / 2$ and $N$ growing, this multiplicity will be positive with probability tending to 1 .

This heuristic does not rule out the possible conspiracy that only very few (about $N^{2 \delta-1}$ ) d's actually appear, each with very high (about $N$ ) multiplicity. But such an argument in reverse leads to another bit of evidence toward (2.15): since the multiplicity of any $d<N$ is at most $N$, we have the elementary lower bound

$$
\# \mathscr{D}_{\mathcal{A}}(N) \geq \frac{1}{N} \# \mathscr{R}_{\mathcal{A}}(N) \stackrel{(2.17)}{\gg} \frac{1}{N} N^{2 \delta_{\mathcal{A}}}=N^{2 \delta_{\mathcal{A}}-1} .
$$

So if $\delta_{\mathcal{A}}>1 / 2$, then the set $\mathscr{D}_{\mathcal{A}}$ already grows at least at a power rate. Furthermore, for any fixed $\varepsilon>0$, one can take some $\mathcal{A}=\mathcal{A}(\varepsilon)$ sufficiently large so that $2 \delta_{\mathcal{A}}-1>$ $1-\varepsilon$. For example, using (2.13), we can take $\mathcal{A}=\{1,2, \ldots, A\}$ where

$$
A>\frac{12}{\pi^{2} \varepsilon}(1+o(1)) \text {. }
$$

Here $o(1) \rightarrow 0$ as $\varepsilon \rightarrow 0$. Hence one can produce $N^{1-\varepsilon}$ points in $\mathscr{D}_{\mathcal{A}}(N)$, which is already substantial progress toward (2.18).

But unfortunately, Hensley's conjecture (2.15), as stated, is false.

Lemma 2.20 (Bourgain and Kontorovich, 2011 [BK11, Lemma 1.19]). The alphabet $\mathcal{A}=\{2,4,6,8,10\}$ has dimension $\delta_{\mathcal{A}}=0.517 \ldots$, which exceeds $1 / 2$, but does not contain every sufficiently large number.

Proof. The dimension can be computed by the Jenkinson-Pollicott algorithm used to establish (2.12). It is an elementary calculation from the definitions to show for this alphabet that every fraction in $\mathscr{R}_{\mathcal{A}}$ is of the form $2 m /(4 n+1)$ or $(4 n+1) /(2 m)$, and so $\mathscr{D}_{\mathcal{A}} \equiv\{0,1,2\}(\bmod 4)$. Hence $\mathscr{D}_{\mathcal{A}}$ does not contain every sufficiently large number.

That is, there can be congruence obstructions, in addition to the condition on dimension. This suggests instead a closer analogy with Hilbert's 11th problem, which asks, What numbers are represented by a given integral (or rational) quadratic form? According to this analogy, we make the following

Definition 2.21. Call $d$ represented by the given alphabet $\mathcal{A}$ if $d \in \mathscr{D}_{\mathcal{A}}$. Also, call $d$ admissible for the alphabet $\mathcal{A}$ if it is everywhere locally represented, meaning that $d \in \mathscr{D}_{\mathcal{A}}(\bmod q)$ for all $q \geq 1$. 
One can then modify Hensley's conjecture to state that, if $\delta_{\mathcal{A}}$ exceeds $1 / 2$ (an Archimedean condition), then every sufficiently large admissible number is represented, akin to Hasse's local-to-global principle.

Remark 2.22. We will explain in $\$ 2.2$ that the alphabet $\mathcal{A}=A=2\{1,2\}$ has no local obstructions, so Hensley's first conjecture (2.9) is still plausible.

Here is some progress toward the conjecture.

Theorem Z (Bourgain and Kontorovich, 2011 [BK11]). Almost every natural number is the denominator of a reduced fraction whose partial quotients are bounded by 50 .

Here "almost every" is in the sense of density: for $\mathcal{A}=\{1,2, \ldots, 50\}$,

$$
\frac{1}{N} \#\left(\mathscr{D}_{\mathcal{A}} \cap[1, N]\right) \rightarrow 1,
$$

as $N \rightarrow \infty$. The proof in fact shows that for any alphabet $\mathcal{A}$ having sufficiently large dimension

$$
\delta_{\mathcal{A}}>\delta_{0},
$$

almost every admissible number is represented, where the value

$$
\delta_{0}=1-5 / 312 \approx 0.98
$$

is sufficient. Using refined versions of Hensley's asymptotic expansion (2.13), the value $A=50$ seems to satisfy (2.23). The reason Theorem $\mathrm{Z}$ needs no mention of admissibility is that any alphabet $\mathcal{A}$ with such a large dimension (2.24) must already contain both 1 and 2; missing even one of these letters will drop the dimension by too much. Hence there are actually no local obstructions in the theorem, cf. Remark 2.22

To explain the source of this progress, we reformulate Zaremba's problem in a way that highlights the role of the hitherto unmentioned "thin orbit" lurking underneath.

2.1. Reformulation. The key to the above progress is the old and elementary observation that

$$
\frac{b}{d}=\left[a_{1}, \ldots, a_{k}\right]
$$

is equivalent to

$$
\left(\begin{array}{ll}
* & b \\
* & d
\end{array}\right)=\left(\begin{array}{cc}
0 & 1 \\
1 & a_{1}
\end{array}\right) \cdots\left(\begin{array}{cc}
0 & 1 \\
1 & a_{k}
\end{array}\right) .
$$

With this observation, it is natural to introduce the semigroup generated by matrices of the above form with partial quotients restricted to the given alphabet. Let

$$
\Gamma=\Gamma_{\mathcal{A}}:=\left\langle\left(\begin{array}{ll}
0 & 1 \\
1 & a
\end{array}\right): a \in \mathcal{A}\right\rangle^{+},
$$

where the superscript "+" denotes generation as a semigroup (no inverse matrices). Then the orbit

$$
\mathcal{O}=\mathcal{O}_{\mathcal{A}}:=\Gamma \cdot \mathbf{v}_{0}
$$


with

$$
\mathbf{v}_{0}=(0,1)^{t}
$$

isolates the set of second columns in $\Gamma$, and from (2.25) is hence in bijection with the set $\mathscr{R}_{\mathcal{A}}$. The "thinness" of the orbit is explained by Hensley's counting statement (2.17), which implies that

$$
\#\{\mathbf{v} \in \mathcal{O}:\|\mathbf{v}\|<N\} \asymp N^{2 \delta_{\mathcal{A}}},
$$

as $N \rightarrow \infty$. If $\mathcal{O}$ consisted of all integer pairs $(b, d)^{t}$, the above count would be replaced by $N^{2}$, ignoring constants. So this is the reason we call $\mathcal{O}$ thin: it contains many fewer points than the ambient set in which it naturally sits.

From (2.25) again, the set $\mathscr{D}_{\mathcal{A}}$ is nothing more than the set of bottom right entries of matrices in $\Gamma_{\mathcal{A}}$. This can be isolated via

$$
\left\langle\mathbf{v}_{0}, \mathcal{O}\right\rangle=\left\langle\mathbf{v}_{0}, \Gamma \cdot \mathbf{v}_{0}\right\rangle=\mathscr{D}_{\mathcal{A}},
$$

where the inner product is the standard one on $\mathbb{R}^{2}$. Thus $d$ is represented if and only if there is a $\gamma \in \Gamma$ so that

$$
d=\left\langle\mathbf{v}_{0}, \gamma \cdot \mathbf{v}_{0}\right\rangle
$$

with $\mathbf{v}_{0}$ given in (2.28).

2.2. Local obstructions. One can now easily understand Remark 2.22, and the source of any potential local obstructions. The key observation, via (2.29), is that to understand $\mathscr{D}_{\mathcal{A}}(\bmod q)$, one needs only to understand the reduction of $\Gamma(\bmod q)$. And the latter can be analyzed by some algebra, namely the so-called strong approximation property; see e.g. Rap12 for a comprehensive survey. As we will see below, this is a property which determines when the reduction $\bmod q$ map is onto. For general algebraic groups this is a deep theory, the first proof [MVW84] using the classification of finite simple groups. But for $\mathrm{SL}_{2}$, the proofs are elementary, see e.g. DSV03.

First observe that $\Gamma$ sits inside the integer points of the algebraic group $\mathrm{GL}_{2}$, meaning that any solution in $\mathbb{Z}$ to the polynomial equation $(a d-b c) m=1$ gives an element $\left(\begin{array}{ll}a & b \\ c & d\end{array}\right) \in \mathrm{GL}_{2}(\mathbb{Z})$, and vice-versa. Actually $\mathrm{GL}_{2}$ does not have strong approximation, (e.g. the determinant in $\mathrm{GL}_{2}(\mathbb{Z})$ can only be \pm 1 , while in $\mathrm{GL}_{2}(\mathbb{Z} / 5 \mathbb{Z})$ it is $1,2,3$ or 4 ; hence the reduction map cannot be onto). So we first pass to $\mathrm{SL}_{2}$, as follows. The generators in (2.26) all have determinant -1 , so the product of any two has determinant +1 . We make these products the generators for a subsemigroup $\tilde{\Gamma}$ of $\Gamma$, that is, set $\tilde{\Gamma}:=\Gamma \cap \mathrm{SL}_{2}$. We recover the original $\Gamma$-orbit $\mathcal{O}$ in (2.27) by a finite union of $\tilde{\Gamma}$-orbits. The limiting Cantor set and its Hausdorff dimension are unaffected.

Then strong approximation says essentially that for $p$ a sufficiently large prime and $q=p^{e}$ any $p$ power, the reduction of $\tilde{\Gamma} \bmod q$ is all of $\mathrm{SL}_{2}(\mathbb{Z} / q \mathbb{Z})$. (It does not matter that $\tilde{\Gamma}$ is only a semigroup; upon reduction $\bmod q$, it becomes a group.) Moreover for ramified primes $p$ (those for which the reduction $\bmod p$ is not onto), the reduction mod sufficiently large powers of $p$ stabilizes after some finite height. This means that there is some power $e_{0}=e_{0}(p, \tilde{\Gamma})$ so that the following holds. For any higher power $e>e_{0}$, if $M \in \mathrm{SL}_{2}\left(\mathbb{Z} / p^{e} \mathbb{Z}\right)$ is such that its reduction is in $\tilde{\Gamma}\left(\bmod p^{e_{0}}\right)$, then $M$ is also in $\tilde{\Gamma}\left(\bmod p^{e}\right)$. (These statements are best made in the language of $p$-adic numbers, which we avoid here.) A key ingredient is that, while $\tilde{\Gamma}$ is some strange subset of $\mathrm{SL}_{2}(\mathbb{Z})$, it is nevertheless Zariski dense in $\mathrm{SL}_{2}$. This 
means that if $P(a, b, c, d)$ is a polynomial which vanishes for every $\left(\begin{array}{ll}a & b \\ c & d\end{array}\right) \in \tilde{\Gamma}$, then $P$ also vanishes on all matrices in $\mathrm{SL}_{2}$ with entries in $\mathbb{C}$.

In the above, "sufficiently large", both for primes $p$ to be unramified and the stabilizing powers $e_{0}$ of ramified primes, can be effectively computed in terms of the generators. Then for an arbitrary modulus $q=p_{1}^{e_{1}} \cdots p_{k}^{e_{k}}$, the reduction $\bmod q$ can be pieced together from those $\bmod p_{j}^{e_{j}}$ using a type of Chinese Remainder Theorem for groups called Goursat's Lemma. This leaves some finite group theory to determine completely the reduction of $\tilde{\Gamma} \bmod$ any $q$, and hence explains all local obstructions via (2.29).

We now leave Zaremba's problem and return to sketch a proof of Theorem $Z$ in 95.

\section{Integral Apollonian gaskets}

Apollonius of Perga (ca. 262-190 BCE) wrote a two-volume book on tangencies, solving in every conceivable configuration the following general problem: Given three circles in the plane, any of which may have radius zero (a point) or infinity (a line), construct a circle tangent to the given ones. The volumes were lost but the statements survived via a survey of the work by Pappus. In the special case when the given three circles are themselves mutually tangent with disjoint points of tangency (Figure 6a), Apollonius proved that

there are exactly two solutions

to his problem (Figure 6b). Adding these new circles to the configuration, one has many other triples of tangent circles, and Apollonius's construction can be applied to them (Figure 6r). Iterating in this way ad infinitum, as apparently was first done in Leibniz's notebook, gives rise to a circle packing, the closure of which has become known in the last century as an Apollonian gasket. We restrict our discussion henceforth to bounded gaskets, such as that illustrated in Figure 1] there the number shown inside a circle is its curvature, that is, one over its radius. Such pictures have received considerable attention recently; see e.g. LMW02, GLM03, GLM05, GLM06a, GLM06b, EL07, Sar07, Sar08, BGS10, KO11, Oh10, BF11, Sar11, Fuc11, FS11, OS12, Vin12, LO12, BK12. We will focus our discussion on the following two problems.

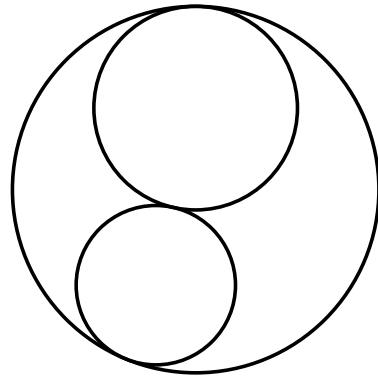

(a) Three mutually tangent circles.

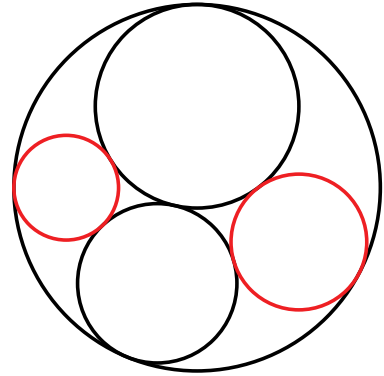

(b) Two more tangent circles.

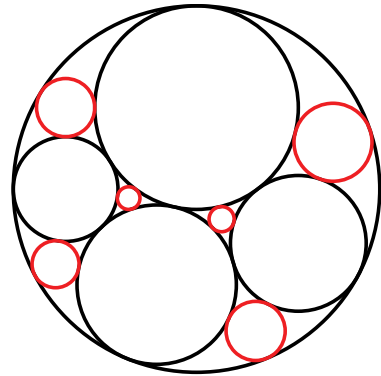

(c) Six more tangent circles. 
(1) The counting problem: For a fixed gasket $\mathscr{G}$, how quickly do the circles shrink, or alternatively, how many circles are there in $\mathscr{G}$ with curvature bounded by a growing parameter $T$ ?

(2) The local-global problem: Suppose $\mathscr{G}$ is furthermore integral, meaning that its circles all have integer curvatures, such as the gasket in Figure 11. How many distinct integers appear up to a growing parameter $N$ ? That is, count curvatures up to $N$, but without multiplicity.

Problem (2) does not yet look like a local-global question, but will soon turn into one. We first address problem (1) in more detail.

\subsection{The counting problem.}

3.1.1. Preliminaries. Some notation: for a typical circle $C$ in a fixed bounded gasket $\mathscr{G}$, let $r(C)$ be its radius and

$$
\kappa(C)=1 / r(C)
$$

its curvature. Let

$$
\mathcal{N}_{\mathscr{G}}(T):=\#\{C \in \mathscr{G}: \kappa(C)<T\}
$$

be the desired counting function. To study this quantity, one might introduce an " $L$-function":

$$
\mathcal{L}_{\mathscr{G}}(s):=\sum_{C \in \mathscr{G}} \frac{1}{\kappa(C)^{s}}=\sum_{C \in \mathscr{G}} r(C)^{s} .
$$

Since the sum of the areas of inside circles in $\mathscr{G}$ yields the area of the bounding circle, the series $\mathcal{L}_{\mathscr{G}}$ converges for $\mathfrak{R e}(s) \geq 2$. It has some abscissa of convergence $\delta$, meaning $\mathcal{L}_{\mathscr{G}}$ converges for $\mathfrak{R e}(s)>\delta$ and diverges for $\mathfrak{R e}(s)<\delta$. Boyd [Boy73] proved that this abscissa $\delta$ is none other than the Hausdorff dimension of the gasket $\mathscr{G}$, as should not be too surprising, comparing (3.3) with the definition (see (2.11)). In fact, Apollonian gaskets are rigid, in the sense that one can be mapped to any other by Möbius transformations. The latter are conformal (angle-preserving) motions of the complex plane, sending $z \mapsto(a z+b) /(c z+d), a d-b c=1$. Hence $\delta$ is a universal constant; McMullen McM98 estimates that

$$
\delta=1.30568 \cdots
$$

From such considerations, Boyd Boy82 was able to conclude that

$$
\frac{\log \mathcal{N}_{\mathscr{G}}(T)}{\log T} \rightarrow \delta
$$

as $T \rightarrow \infty$.

To refine this crude estimate to an asymptotic formula for $\mathcal{N}_{\mathscr{G}}(T)$, the author and Oh KO11] established a "spectral interpretation" for $\mathcal{L} \mathscr{G}$, proving

$$
\mathcal{N}_{\mathscr{G}}(T) \sim c \cdot T^{\delta}
$$

for some $c=c(\mathscr{G})>0$, as $T \rightarrow \infty$. (This asymptotic was recently refined further in Vinogradov's thesis Vin12 and independently by Lee and Oh [LO12, giving lower order error terms.) The remainder of this subsection is devoted to explaining this spectral interpretation and highlighting some of the ideas going into the proof of (3.5). 


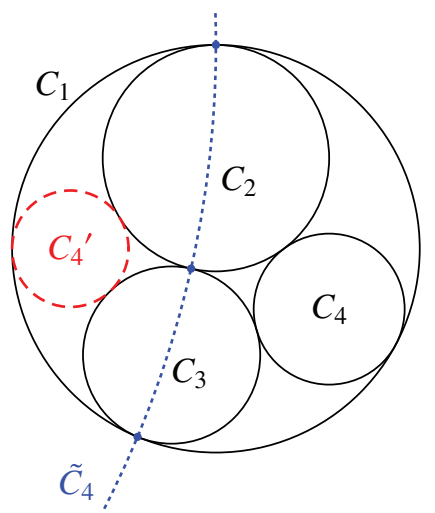

FiguRE 7. Generation from a root quadruple.

3.1.2. Root quadruples and generation by reflection. It is easy to see GLM03, p. 14] that each such gasket $\mathscr{G}$ contains a root configuration $\mathcal{C}=\mathcal{C}(\mathscr{G}):=\left(C_{1}, C_{2}, C_{3}, C_{4}\right)$ of four largest mutually tangent circles in $\mathscr{G}$. Let

$$
\mathbf{v}_{0}=\mathbf{v}_{0}(\mathscr{G})=\left(\kappa_{1}, \kappa_{2}, \kappa_{3}, \kappa_{4}\right)^{t}
$$

with $\kappa_{j}=\kappa\left(C_{j}\right)$ be the root quadruple of corresponding curvatures. The bounding circle, being internally tangent to the others, is given the opposite orientation to make all interiors disjoint; this is accounted for by giving it negative curvature. For example, in Figure 1, the root quadruple is

$$
\mathbf{v}_{0}=(-10,18,23,27)^{t}
$$

where the bounding circle has radius $1 / 10$.

Three tangent circles, say $C_{1}, C_{2}, C_{3}$ have three points of tangency, and they determine a dual circle $\tilde{C}_{4}$ passing through these points; see Figure 7 . Thus the root configuration $\mathcal{C}$ determines a dual configuration $\tilde{\mathcal{C}}=\left(\tilde{C}_{1}, \tilde{C}_{2}, \tilde{C}_{3}, \tilde{C}_{4}\right)$ of four mutually tangent circles, orthogonal to those in $\mathcal{C}$; see Figure 8, Reflection through $\tilde{C}_{4}$ fixes $C_{1}, C_{2}$, and $C_{3}$, and sends $C_{4}$ to $C_{4}^{\prime}$, the other solution to Apollonius's problem (3.1); see Figure 7 . Starting with the root configuration, repeated reflections through the dual circles give the whole circle packing.

3.1.3. Hyperbolic space and the group $\mathcal{A}$. Following Poincaré, we extend these circle reflections to the hyperbolic upper half-space,

$$
\mathbb{H}^{3}:=\left\{\left(x_{1}, x_{2}, y\right): x_{1}, x_{2} \in \mathbb{R}, y>0\right\},
$$

replacing the action of the dual circle $\tilde{C}_{j}$ by a reflection through a (hemi)sphere $\mathfrak{s}_{j}$ whose equator is $\tilde{C}_{j}$ (with $j=1, \ldots, 4$ ). We abuse notation, writing $\mathfrak{s}_{j}$ for both the hemisphere and the conformal map reflecting through $\mathfrak{s}_{j}$. The group

$$
\mathcal{A}:=\left\langle\mathfrak{s}_{1}, \mathfrak{s}_{2}, \mathfrak{s}_{3}, \mathfrak{s}_{4}\right\rangle<\operatorname{Isom}\left(\mathbb{H}^{3}\right),
$$

generated by these reflections, acts discretely on $\mathbb{H}^{3}$; it is a so-called Schottky group, in that the four generating spheres have disjoint interiors.

The $\mathcal{A}$-orbit of any fixed basepoint $p_{0} \in \mathbb{H}^{3}$ has a limit set in the boundary $\partial \mathbb{H}^{3}$, which is easily seen to be the original gasket; see Figure 9. A fundamental domain 


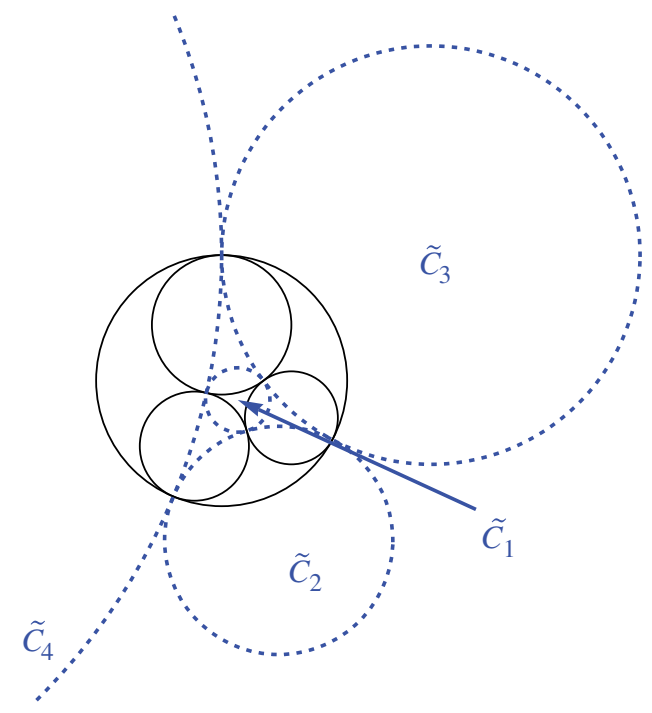

FiguRE 8. Root and dual configurations.

for an action is a region

$$
\Omega \subset \mathbb{H}^{3},
$$

so that any point in $\mathbb{H}^{3}$ can be sent to $\Omega$ in an essentially unique way; for the action of $\mathcal{A}$, one can take $\Omega$ to be the exterior of the four hemispheres.

To see this, observe that if a point $p=\left(x_{1}, x_{2}, y\right) \in \mathbb{H}^{3}$ is inside one of the spheres $\mathfrak{s}_{j}$, then its reflection $\mathfrak{s}_{j}(p)$ is outside of $\mathfrak{s}_{j}$ and has a strictly larger $y$-value. This does not guarantee that $\mathfrak{s}_{j}(p)$ is outside all of the other spheres, but if it is inside some $\mathfrak{s}_{k}$, then reflection through $\mathfrak{s}_{k}$ will again have even higher $y$-value. This procedure must halt after finitely many iterations, since the only limit points of $\mathcal{A}$ are in the boundary $\partial \mathbb{H}^{3}$, where $y=0$. And it halts only when the image is outside of the four geodesic hemispheres. Uniqueness follows since any reflection $\mathfrak{s}_{j}$ takes a point in $\Omega$ to a point inside $\mathfrak{s}_{j}$, that is, not in $\Omega$.

Two facts are evident from the above: first of all, $\mathcal{A}$ is geometrically finite, meaning it has a fundamental domain bounded by a finite number (here it is four) of geodesi 2 hemispheres; on the other hand, $\mathcal{A}$ has infinite covolume, that is, any fundamental domain has infinite volume with respect to the hyperbolic measure

$$
y^{-3} d x_{1} d x_{2} d y
$$

in the coordinates (3.8). Note moreover that $\mathcal{A}$ has the structure of a Coxeter group, being free save the relations $\mathfrak{s}_{j}^{2}=I$ for the generators. It is also the symmetry group of all Möbius transformations fixing $\mathscr{G}$.

3.1.4. Descartes' Circle Theorem and integral gaskets. Next we need an observation attributed to Descartes in the year 1643 ([Des01, pp. 37-50], though his proof had a gap Cox68]), that a quadruple $\mathbf{v}=\left(b_{1}, b_{2}, b_{3}, b_{4}\right)^{t}$ of signed curvatures of four

\footnotetext{
${ }^{2} \mathrm{~A}$ geodesic in hyperbolic space is a straight vertical line or a semicircle orthogonal to the boundary $\partial \mathbb{H}^{3}$.
} 


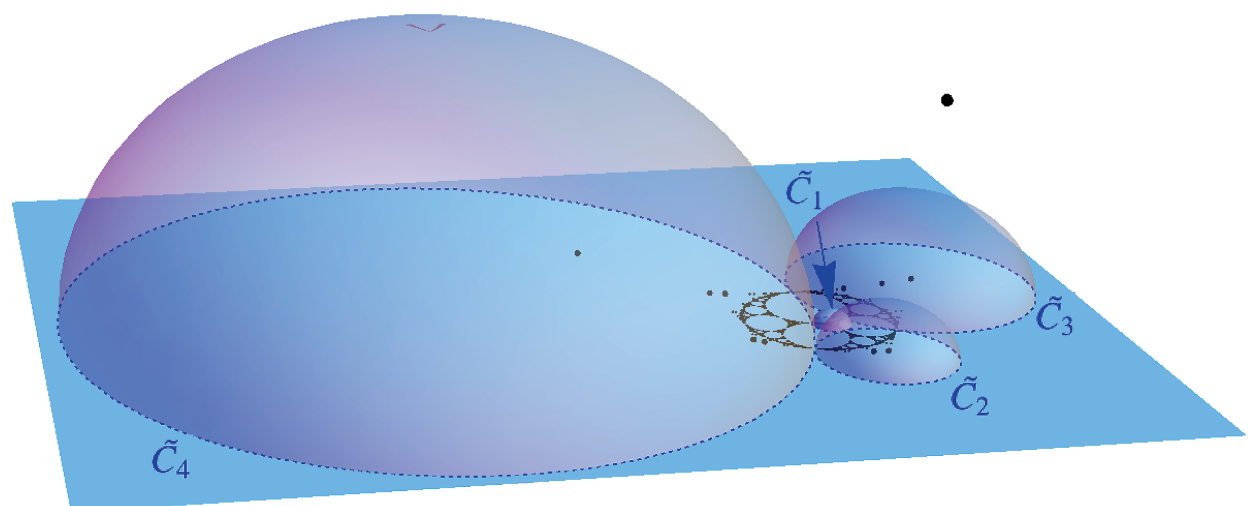

Figure 9. Poincaré extension: an $\mathcal{A}$-orbit in $\mathbb{H}^{3}$.

mutually tangent circles lies on the cone

$$
Q(\mathbf{v})=0,
$$

where $Q$ is the so-called "Descartes quadratic form"

$$
Q(\mathbf{v}):=2\left(\kappa_{1}^{2}+\kappa_{2}^{2}+\kappa_{3}^{2}+\kappa_{4}^{2}\right)-\left(\kappa_{1}+\kappa_{2}+\kappa_{3}+\kappa_{4}\right)^{2} .
$$

By a real linear change of variables, $Q$ can be diagonalized to the form

$$
x^{2}+y^{2}+z^{2}-w^{2},
$$

that is, it has signature $(3,1)$. Arguably the most beautiful formulation of Descartes' Theorem (rediscovered on many separate occasions) is the following excerpt from Soddy's 1936 Nature poem Sod36]:

\footnotetext{
Four circles to the kissing come. / The smaller are the bender. /

The bend is just the inverse of / The distance from the center. /

Though their intrigue left Euclid dumb / There's now no need for rule of thumb. /

Since zero bend's a dead straight line / And concave bends have minus sign, /

The sum of the squares of all four bends / Is half the square of their sum.
}

If $\kappa_{1}, \kappa_{2}$ and $\kappa_{3}$ are given, then (3.11) is a quadratic equation in $\kappa_{4}$ with two solutions, $\kappa_{4}$ and $\kappa_{4}^{\prime}$, say; this is an algebraic proof of Apollonius's theorem (3.1). It is then an elementary exercise to see that

$$
\kappa_{4}+\kappa_{4}^{\prime}=2\left(\kappa_{1}+\kappa_{2}+\kappa_{3}\right) .
$$

In other words, if the quadruple $\left(\kappa_{1}, \kappa_{2}, \kappa_{3}, \kappa_{4}\right)^{t}$ is given, then one obtains the quadruple with $\kappa_{4}$ replaced by $\kappa_{4}^{\prime}$ via a linear action:

$$
\left(\begin{array}{cccc}
1 & & & \\
& 1 & & \\
2 & 2 & 2 & -1
\end{array}\right) \cdot\left(\begin{array}{l}
\kappa_{1} \\
\kappa_{2} \\
\kappa_{3} \\
\kappa_{4}
\end{array}\right)=\left(\begin{array}{l}
\kappa_{1} \\
\kappa_{2} \\
\kappa_{3} \\
\kappa_{4}^{\prime}
\end{array}\right) .
$$

Hence we have given an algebraic realization to the geometric action of $\tilde{C}_{4}\left(\right.$ or $\left.\mathfrak{s}_{4}\right)$ on the root quadruple; see again Figure 7 Call the above $4 \times 4$ matrix $S_{4}$. Of course one could also send other $\kappa_{j}$ to $\kappa_{j}^{\prime}$ keeping the three complementary curvatures 
fixed, via the matrices

$$
S_{1}=\left(\begin{array}{cccc}
-1 & 2 & 2 & 2 \\
& 1 & & \\
& & 1 & \\
& & & 1
\end{array}\right), S_{2}=\left(\begin{array}{llll}
1 & & & \\
2 & -1 & 2 & 2 \\
& & 1 & \\
& & & 1
\end{array}\right), S_{3}=\left(\begin{array}{llll}
1 & & & \\
& 1 & & \\
2 & 2 & -1 & 2 \\
& & & 1
\end{array}\right)
$$

Moreover one can iterate these actions, so we introduce the so-called Apollonian group $\Gamma$, isomorphic to $\mathcal{A}$, generated by the $S_{j}$,

$$
\Gamma:=\left\langle S_{1}, S_{2}, S_{3}, S_{4}\right\rangle .
$$

Then the orbit

$$
\mathcal{O}:=\Gamma \cdot \mathbf{v}_{0}
$$

of the root quadruple $\mathbf{v}_{0}$ under the Apollonian group $\Gamma$ consists of all quadruples corresponding to curvatures of four mutually tangent circles in the gasket $\mathscr{G}$. We can now explain the integrality of all curvatures in Figure 1 the group $\Gamma$ has only integer matrices, so if the root quadruple $\mathbf{v}_{0}$ (or for that matter any four curvatures of mutually tangent circles in $\mathscr{G}$ ) is integral, then all curvatures in $\mathscr{G}$ are integers! This fact seems to have been first observed by Soddy [Sod37].

3.1.5. Reformulating the counting statement and the thin orbit. We note, moreover, that starting with $\mathbf{v}_{0}$, any new circle generated by a reflection is the smallest in its configuration, and hence has largest curvature. That is, for $\mathbf{v}=\gamma \cdot \mathbf{v}_{0} \in \mathcal{O}$, writing $\gamma \in \Gamma$ as a reduced word in the generators $\gamma=S_{i_{k}} \cdots S_{i_{1}}$, the last multiplication by $S_{i_{k}}$ changes one entry, which is the largest entry in $\mathbf{v}$. Hence, setting $\|\mathbf{v}\|_{\infty}$ to be the max-norm and for $T$ large, we can rewrite $\mathcal{N}_{\mathscr{G}}(T)$ in (3.2) as

$$
\mathcal{N}_{\mathscr{G}}(T)=4+\#\left\{\mathbf{v} \in \mathcal{O}: \mathbf{v} \neq \mathbf{v}_{0},\|\mathbf{v}\|_{\infty}<T\right\} .
$$

Here the first " 4 " accounts for the root quadruple $\mathbf{v}_{0}$.

We have thus converted the circle counting problem into something seemingly more tractable: the counting problem for a $\Gamma$-orbit. That said, we clearly need a better understanding of the group $\Gamma$. Returning to the Descartes form $Q$ in (3.12), we have by construction (and one can check directly) that for each $j=1, \ldots, 4$,

$$
Q\left(S_{j} \cdot \mathbf{v}\right)=Q(\mathbf{v})
$$

for any $\mathbf{v}$. That is, each generator $S_{j}$ lies in the so-called orthogonal group preserving the quadratic form $Q$,

$$
O_{Q}:=\left\{g \in \mathrm{GL}_{4}: Q(g \cdot \mathbf{v})=Q(\mathbf{v}), \forall \mathbf{v}\right\} .
$$

Hence $\Gamma$ also sits inside $O_{Q}$, and moreover inside $O_{Q}(\mathbb{Z})$, the group of matrices in $O_{Q}$ with integer entries. The latter is a well understood algebraic group, again meaning that any solution to a certain set of polynomial equations gives an element in $O_{Q}$, and vice-versa. But $\Gamma$ is quite a mysterious group, in particular having infinite index in $O_{Q}(\mathbb{Z})$ (this fact is equivalent to $\mathcal{A}$ having infinite covolume). It is also worth noting here that the general membership problem in a group is known to be undecidable Nov55, so presenting a matrix group via its generators leaves much to be desired 3

Just as in Zaremba's problem, we can now again call this orbit $\mathcal{O}$ thin; indeed, for the counting problem with $\Gamma$ replaced by the full group $O_{Q}(\mathbb{Z})$ (which is an example

\footnotetext{
${ }^{3}$ That said, for our particular group $\Gamma$, one can use a reduction algorithm to root quadruples to determine membership.
} 
of what is called an "arithmetic lattice"), standard arguments in automorphic forms or ergodic theory DRS93, EM93, show that

$$
\#\left\{\mathbf{v} \in O_{Q}(\mathbb{Z}) \cdot \mathbf{v}_{0}:\|\mathbf{v}\|_{\infty}<T\right\} \sim c T^{2}, \quad \text { as } T \rightarrow \infty,
$$

for some $c>0$. So comparing (3.17) to (3.16), (3.5) and (3.4), where the power drops from $T^{2}$ to $T^{\delta}$ with $\delta<2$, we see that the $\Gamma$ orbit is quite degenerate, having many fewer points.

3.1.6. Sketch of the counting statement. Finally, we explain the aforementioned spectral interpretation by first giving an analogous elementary example of a counting statement in another discrete group: the integers. Let us spectrally count the number of integers of size at most $T$,

$$
\mathcal{N}_{\mathbb{Z}}(T):=\#\{n \in \mathbb{Z}:|n|<T\} .
$$

Of course this is a trivial problem,

$$
\mathcal{N}_{\mathbb{Z}}(T)=\lfloor 2 T+1\rfloor=2 T+O(1),
$$

but it will be instructive to analyze it by harmonic analysis. To this end, let

$$
f(x):=\mathbf{1}_{\{|x|<1\}},
$$

where $\mathbf{1}_{\{\cdot\}}$ is the indicator function. Scale $f$ to

$$
f_{T}(x):=f(x / T)=\mathbf{1}_{\{|x|<T\}},
$$

and periodize it with respect to the discrete group $\mathbb{Z}$,

$$
F_{T}(x):=\sum_{n \in \mathbb{Z}} f_{T}(n+x) .
$$

Then we have

$$
F_{T}(0)=\sum_{n \in \mathbb{Z}} \mathbf{1}_{\{|n|<T\}}=\mathcal{N}_{\mathbb{Z}}(T) .
$$

By construction, $F_{T}(x)=F_{T}(x+1)$, that is, it takes values on the circle $X:=\mathbb{Z} \backslash \mathbb{R}$, and is square-integrable, $F_{T} \in L^{2}(X)$. The Laplace operator

$$
\Delta:=-\operatorname{div} \circ \operatorname{grad}=-\frac{\partial^{2}}{\partial x^{2}}
$$

on smooth functions can be extended to act on the whole Hilbert space $L^{2}(X)$ and is self-adjoint and positive definite (by our choice of sign) with respect to the standard inner product

$$
\langle F, G\rangle=\int_{X} F(x) \bar{G}(x) d x .
$$

(Proof: partial integration.) Its spectrum $\operatorname{Spec}(\Delta)$ is just the set of its eigenvalues, with multiplicity. Elementary Fourier analysis shows that eigenfunctions of $\Delta$ invariant under $\mathbb{Z}$-translations are scalar multiples of

$$
\varphi_{m}: x \mapsto e^{2 \pi i m x}
$$

for $m \in \mathbb{Z}$. This function has Laplace eigenvalue

$$
\lambda_{m}=4 \pi^{2} m^{2},
$$


and hence these numbers $\lambda_{m}$ completely exhaust the spectrum (they have multiplicity two, except when $m=0$ ). Expanding spectrally gives

$$
F_{T}(x)=\sum_{\lambda_{m} \in \operatorname{Spec}(\Delta)}\left\langle F_{T}, \varphi_{m}\right\rangle \varphi_{m}(x),
$$

where equality is in the $L^{2}$-sense. (Note that the $\varphi_{m}$ are already scaled to have unit $L^{2}$-norm.) The bottom of the spectrum $\lambda_{0}=0$ corresponds to the constant function $\varphi_{0}(x)=1$, and contributes the entire "main term" in (3.18) to (3.21):

$$
\left\langle F_{T}, \varphi_{0}\right\rangle \cdot \varphi_{0}=\left(\int_{\mathbb{Z} \backslash \mathbb{R}} \sum_{n \in \mathbb{Z}} f_{T}(n+x) \cdot 1 d x\right) \cdot 1=T \int_{\mathbb{R}} f(x) d x=2 T,
$$

after inserting (3.19), a change of variables, and "unfolding" $\int_{\mathbb{Z} \backslash \mathbb{R}} \sum_{\mathbb{Z}}$ to just $\int_{\mathbb{R}}$. That said, the equality (3.21) is in the $L^{2}$ sense, not pointwise (we cannot evaluate (3.21) at the point $x=0$, as needed in (3.20). Moreover, the rest of the spectrum in (3.21), if bounded in absolute value,

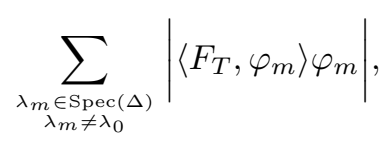

does not converge, the $m$ th term being of size $1 / m$. (Exercise.) But there are standard methods (smoothing and later unsmoothing) which overcome these technical irritants.

A version of the above works with the Apollonian group $\Gamma$ in place of $\mathbb{Z}$, once one overcomes a number of further technical obstructions. The reader may wish to omit the following paragraph on the first pass; it is not essential to the sequel.

We now need non-Abelian harmonic analysis on the space $L^{2}(X)$ with

$$
X:=\mathcal{A} \backslash \mathbb{H}^{3},
$$

the hyperbolic 3-fold in Figure 9. The (positive definite) hyperbolic Laplacian is

$$
\Delta=-y^{2}\left(\frac{\partial^{2}}{\partial x_{1}^{2}}+\frac{\partial^{2}}{\partial x_{2}^{2}}+\frac{\partial^{2}}{\partial y^{2}}\right)+y \frac{\partial}{\partial y}
$$

in the coordinates (3.8). The spectrum in this setting, as studied by Lax and Phillips LP82, has both continuous and discrete components (though only a finite number of the latter). As $X$ has infinite volume, the constant function is no longer squareintegrable, and the bottom eigenvalue $\lambda_{0}$ is strictly positive. A beautiful result in Patterson-Sullivan theory [Pat76, Sul84] relates this eigenvalue to the Hausdorff dimension of the limiting gasket $\mathscr{G}$, namely

$$
\lambda_{0}=\delta(2-\delta) .
$$

The corresponding base eigenfunction $\varphi_{0}$ replaces the role of the constant function. Here we have used crucially that $\mathcal{A}$ is geometrically finite and that $\delta>1$; see (3.4). Even this is insufficient: because of the non-Euclidean norm $\|\cdot\|_{\infty}$ in (3.16), one must work not on $X$ but its unit tangent bundle $Y:=T^{1}(X)$. And moreover we do not know how to handle the continuous spectrum directly, applying instead general results in the representation theory of semisimple groups about ergodic properties of flows on $Y$. At this point, we will not say more about the proof, inviting the interested reader to consult the original references [KO11, Vin12, LO12]. 
3.2. The local-global problem. Assume now that $\mathscr{G}$ is not only bounded but also integral (recall that this means it has only integer curvatures). If the curvatures are all even, say, then we can stretch the gasket by a factor of two, doubling the radii and halving the (still integral) curvatures. In this way, we can rescale an integral gasket to make it primitive, meaning that there is no number other than \pm 1 dividing all of the curvatures. In fact, all of the salient features of the problem persist if we fix $\mathscr{G}$ to be the packing shown in Figure 1 and we do so henceforth. Recall the problem we wish to now address is, How many curvatures are there up to some parameter $N$, counting without multiplicity, that is, counting only distinct curvatures?

First some more notation. Let $\mathscr{K}=\mathscr{K}(\mathscr{G})$ be the set of all curvatures of circles $C$ in the gasket $\mathscr{G}$,

$$
\mathscr{K}:=\{n \in \mathbb{Z}: \exists C \in \mathscr{G} \text { with } \kappa(C)=n\},
$$

and call $n$ represented if $n \in \mathscr{K}$. Staring at Figure 1 for a moment or two, one might observe that every curvature in our $\mathscr{G}$ is

$$
\equiv 2,3,6,11,14,15,18 \text {, or } 23(\bmod 24) \text {. }
$$

These are the local obstructions for $\mathscr{G}$; accordingly, we call $n$ admissible if it satisfies (3.22), and we set $\mathscr{A}=\mathscr{A}(\mathscr{G})$ to be the set of admissible numbers. In general, one calls $n$ admissible if, as before, it is everywhere locally represented,

$$
n \in \mathscr{K}(\bmod q), \forall q \geq 1 .
$$

It cannot be the case that $\mathscr{A}=\mathscr{K}$, since, for example, $n=15$ is admissible, but a circle of radius $1 / 15$ does not appear in our gasket. Nevertheless, as in Zaremba's problem, we have the following

Conjecture A. Every sufficiently large admissible number is the curvature of some circle in $\mathscr{G}$.

This conjecture is stated by Graham, Lagarias, Mallows, Wilks, and Yan [GLM03, p. 37] in the first of a lovely series of papers on Apollonian gaskets and generalizations. They observe empirically that congruence obstructions for any integral gasket seem to be to the modulus 24, and this is completely clarified (as we explain below) by Fuchs [Fuc11] in her thesis. Further convincing numerical evidence toward the conjecture is given in Fuch and Sanden [FS11. Here is some recent progress.

Theorem A (Bourgain, and Kontorovich, 2012 [BK12]). Almost every admissible number is the curvature of some circle in $\mathscr{G}$.

Again, "almost every" is in the sense of density, that

$$
\frac{\#(\mathscr{K} \cap[1, N])}{\#(\mathscr{A} \cap[1, N])} \rightarrow 1,
$$

as $N \rightarrow \infty$. It follows from the congruence restrictions (3.22) that for $N$ large, $\#(\mathscr{A} \cap[1, N])$ is about $N / 3$ (there are eight admissible residue classes mod 24 ), so (3.24) is equivalent to

$$
\#(\mathscr{K} \cap[1, N]) \sim \frac{N}{3} .
$$


Some history on this problem: Graham et al. GLM03 already made the first progress, proving that

$$
\#(\mathscr{K} \cap[1, N]) \gg N^{1 / 2} .
$$

Then Sarnak [Sar07] showed

$$
\#(\mathscr{K} \cap[1, N]) \gg \frac{N}{\sqrt{\log N}},
$$

before Bourgain and Fuchs [BF11] settled the so-called Positive Density Conjecture, that

$$
\#(\mathscr{K} \cap[1, N]) \gg N
$$

A key observation in the proof of Theorem $\mathrm{A}$ is that the problem is nearly identical to Zaremba's, in the following sense. Recall from (3.15) that the orbit $\mathcal{O}=\Gamma \cdot \mathbf{v}_{0}$ of the root quadruple $\mathbf{v}_{0}$ under the Apollonian group $\Gamma$ contains all quadruples of curvatures, and in particular its entries consist of all curvatures in $\mathscr{G}$. Hence the set $\mathscr{K}$ of all curvatures is simply the finite union of sets of the form

$$
\left\langle\mathbf{w}_{0}, \mathcal{O}\right\rangle=\left\langle\mathbf{w}_{0}, \Gamma \cdot \mathbf{v}_{0}\right\rangle,
$$

as $\mathbf{w}_{0}$ ranges through the standard basis vectors

$$
\mathbf{e}_{1}=(1,0,0,0)^{t}, \ldots, \mathbf{e}_{4}=(0,0,0,1)^{t},
$$

each picking off one entry of $\mathcal{O}$. A heuristic analogy between Zaremba and the Apollonian problem is actually already given in [GLM03, p. 37], but it is crucial for us that both problems are exactly of the form (3.28); compare to (2.29). That is, $n$ is represented if and only if there is a $\gamma$ in the Apollonian group $\Gamma$ and some $\mathbf{w}_{0} \in\left\{\mathbf{e}_{1}, \ldots, \mathbf{e}_{4}\right\}$ so that

$$
n=\left\langle\mathbf{w}_{0}, \gamma \cdot \mathbf{v}_{0}\right\rangle .
$$

Before saying more about the proof of Theorem $\mathrm{A}$ we first discuss admissibility in greater detail.

3.2.1. Local obstructions. Through (3.28), the admissibility condition (3.23) is again reduced to the study of the projection of $\Gamma$ modulo $q$. An important feature here is that, as in the Zaremba case, the group $\Gamma$ is Zariski dense in $O_{Q}$. Recall that this means if $P(\gamma)$ is a polynomial in the entries of a $4 \times 4$ matrix $\gamma$ which vanishes for every $\gamma \in \Gamma$, then $P$ also vanishes on all complex matrices in $O_{Q}$.

We would like again to exploit strong approximation, but neither $O_{Q}$ nor its orientation-preserving subgroup $\mathrm{SO}_{Q}:=O_{Q} \cap \mathrm{SL}_{4}$ have this property (being not even connected). But there is a standard method of applying strong approximation anyway, by first passing to a certain cover, as we now describe.

From the theory of rational quadratic forms Cas78, special orthogonal groups are covered by so-called spin groups, and it is a pleasant accident that, since $Q$ has signature $(3,1)$, the spin group of $\mathrm{SO}_{Q}(\mathbb{R})$ is isomorphic to $\mathrm{SL}_{2}(\mathbb{C})$. Let us explain this covering map. The formulae are nicer if we first change variables (over $\mathbb{Q}$ ) from our quadratic form $Q$ to the equivalent form

$$
\tilde{Q}(x, y, z, w):=x w+y^{2}+z^{2} .
$$

Observe that the matrix

$$
M:=\left(\begin{array}{cc}
-x & y+i z \\
y-i z & w
\end{array}\right)
$$


has determinant equal to $-\tilde{Q}$ and is Hermitian, that is, fixed under transposeconjugation. The group $\mathrm{SL}_{2}(\mathbb{C})$, consisting of $2 \times 2$ complex matrices of determinant one, acts on $M$ by

$$
\mathrm{SL}_{2}(\mathbb{C}) \ni g: M \mapsto g \cdot M \cdot \bar{g}^{t}=: M^{\prime}=\left(\begin{array}{cc}
-x^{\prime} & y^{\prime}+i z^{\prime} \\
y^{\prime}-i z^{\prime} & w^{\prime}
\end{array}\right),
$$

with $M^{\prime}$ also Hermitian and of determinant $-\tilde{Q}$. Then it is easy to see that $\left(x^{\prime}, y^{\prime}, z^{\prime}, w^{\prime}\right)^{t}$ is a linear change of variables from $(x, y, z, w)^{t}$, via left multiplication by a matrix whose entries are quadratic in the entries of $g$. Explicitly, if

$$
g=\left(\begin{array}{cc}
a+\alpha i & b+\beta i \\
c+\gamma i & d+\delta i
\end{array}\right)
$$

then the change of variables matrix is

$$
\frac{1}{|\operatorname{det}(g)|^{2}}\left(\begin{array}{cccc}
a^{2}+\alpha^{2} & 2(a c+\alpha \gamma) & 2(c \alpha-a \gamma) & -c^{2}-\gamma^{2} \\
a b+\alpha \beta & b c+a d+\beta \gamma+\alpha \delta & d \alpha+c \beta-b \gamma-a \delta & -c d-\gamma \delta \\
a \beta-b \alpha & -d \alpha+c \beta-b \gamma+a \delta & -b c+a d-\beta \gamma+\alpha \delta & d \gamma-c \delta \\
-b^{2}-\beta^{2} & -2(b d+\beta \delta) & 2(b \delta-d \beta) & d^{2}+\delta^{2}
\end{array}\right)
$$

Let $\tilde{\rho}$ be the (rational) map from $\mathrm{SL}_{2}(\mathbb{C})$ to $\mathrm{GL}_{4}(\mathbb{R})$, sending (3.30) to (3.31); then by construction (again one can verify directly) the image is in $\mathrm{SO}_{\tilde{Q}}(\mathbb{R})$. (Some minor technical points: Being quadratic in the entries, $\tilde{\rho}$ is a double cover, with $\pm I$ having the same image. Moreover, $\mathrm{SL}_{2}(\mathbb{C})$ is connected while $\mathrm{SO}_{\tilde{Q}}(\mathbb{R})$ has two connected components, so $\tilde{\rho}$ only maps onto the identity component $\mathrm{SO}_{\tilde{Q}}^{\circ}$.) Then changing variables from $\tilde{Q}$ back to the Descartes form $Q$ by a conjugation, one gets the desired map

$$
\rho: \mathrm{SL}_{2}(\mathbb{C}) \rightarrow \mathrm{SO}_{Q}(\mathbb{R}) .
$$

It is straightforward then to compute the pullback of $\Gamma \cap \mathrm{SO}_{Q}$ under $\rho$ (see GLM05, Fuc11]), the answer being the following

Lemma 3.32. There $i \unlhd^{4}$ a homomorphism $\rho: \mathrm{SL}_{2}(\mathbb{C}) \rightarrow \mathrm{SO}_{Q}(\mathbb{R})$ so that the group $\tilde{\Gamma}:=\rho^{-1}\left(\Gamma \cap \mathrm{SO}_{Q}\right)$ sits in $\mathrm{SL}_{2}(\mathbb{Z}[i])$ and is generated by

$$
\tilde{\Gamma}=\left\langle \pm\left(\begin{array}{ll}
1 & 2 \\
0 & 1
\end{array}\right), \pm\left(\begin{array}{ll}
1 & 0 \\
2 & 1
\end{array}\right), \pm\left(\begin{array}{cc}
1+2 i & -2 \\
-2 & 1-2 i
\end{array}\right)\right\rangle .
$$

Moreover, recalling the generators $S_{j}$ for $\Gamma$ in (3.13), one can arrange $\rho$ so that $\rho:\left(\begin{array}{ll}1 & 2 \\ 0 & 1\end{array}\right) \mapsto S_{2} S_{3}$, and $\rho:\left(\begin{array}{ll}1 & 0 \\ 2 & 1\end{array}\right) \mapsto S_{4} S_{3}$.

In fact, we have just realized a conjugate of the group $\mathcal{A}$ (or rather its indextwo orientation-preserving subgroup) explicitly in terms of matrices in $\operatorname{PSL}(2, \mathbb{C}) \cong$ Isom $^{+}\left(\mathbb{H}^{3}\right)$.

From here, one follows the strategy outlined in $\$ 2.2$ Fuchs [Fuc11 proved an explicit version of strong approximation for $\tilde{\Gamma}<\mathrm{SL}_{2}(\mathbb{Z}[i]$ ) (one considers reduction mod principal ideals $(q)$ ) via Goursat's Lemma, some finite group theory, and other ingredients, enabling her to determine completely the reduction of $\Gamma$ modulo any $q$, and hence explaining all local obstructions. The answer is that all primes other than 2 and 3 are unramified, meaning, as in 2.2 , that for $(q, 6)=1$,

$$
\Gamma \cap \mathrm{SO}_{Q}(\bmod q)=\mathrm{SO}_{Q}(\mathbb{Z} / q \mathbb{Z}) .
$$

\footnotetext{
${ }^{4}$ And one can easily write it down explicitly: it is a conjugate of (3.31), but much messier and not particularly enlightening. We spare the reader.
} 
Recall again that the right-hand side above is a well understood group. And moreover, the prime 2 stabilizes (with the same meaning as $₫ 2.2$ ) at the power $e_{0}(2)=3$, that is at 8 , and the prime 3 stabilizes immediately at $e_{0}(3)=1$. Then reducing $\Gamma$ modulo $2^{3} \cdot 3=24$, one obtains some explicit finite group, and looking at all the values of (3.28) for the given root quadruple $\mathbf{v}_{0}(\mathscr{G})$, one immediately sees all admissible residue classes.

3.2.2. Partial progress. Lemma 3.32 can already be quite useful; in particular, it easily implies (3.25) and (3.26), as follows.

The Apollonian group $\Gamma$ contains the matrix $S_{4} S_{3}$, which by Lemma 3.32 is the image under $\rho$ of $\left(\begin{array}{ll}1 & 0 \\ 2 & 1\end{array}\right)$. The latter (and hence the former) is a unipotent matrix, meaning that all its eigenvalues are equal to 1 . These have the important property that they grow only polynomially under exponentiation; in particular, $\left(\begin{array}{cc}1 & 0 \\ 2 & 1\end{array}\right)^{k}=\left(\begin{array}{cc}1 & 0 \\ 2 k & 1\end{array}\right)$, and one can check directly from the definitions (3.13) that

$$
\left(S_{4} S_{3}\right)^{k}=\left(\begin{array}{cccc}
1 & 0 & 0 & 0 \\
0 & 1 & 0 & 0 \\
4 k^{2}-2 k & 4 k^{2}-2 k & 1-2 k & 2 k \\
4 k^{2}+2 k & 4 k^{2}+2 k & -2 k & 2 k+1
\end{array}\right) .
$$

Put the above matrix into (3.29) with the root quadruple $\mathbf{v}_{0}$ for our fixed gasket from (3.7), and take $\mathbf{w}_{0}=\mathbf{e}_{4}$, say. Then for any $k \in \mathbb{Z}$, the number

$$
\left\langle\mathbf{e}_{4},\left(S_{4} S_{3}\right)^{k} \cdot \mathbf{v}_{0}\right\rangle=32 k^{2}+24 k+27
$$

is represented. That is, the set of represented numbers contains the values of this quadratic polynomial. From this observation, made in GLM03, it is immediate that (3.25) holds. Geometrically, these curvatures correspond to circles in the packing tangent to $C_{1}$ and $C_{2}$, since these are fixed by the corresponding reflections through $\tilde{C}_{4}$ and $\tilde{C}_{3}$. For example, the values $k=-2,-1,0,1,2$ in (3.34) give curvatures $107,35,27,83,203$, respectively. These are visible in Figure 1 they are all tangent to the circles of curvature -10 (the bounding circle) and 18, skipping every other such circle. Using $\mathbf{w}_{0}=\mathbf{e}_{3}$ instead of $\mathbf{e}_{4}$ in (3.34) gives the polynomial $32 k^{2}-8 k+23$, the values of which correspond to the skipped circles.

To prove (3.26), we make the following observation, due to Sarnak Sar07. It is well known that the matrices $\pm\left(\begin{array}{ll}1 & 0 \\ 2 & 1\end{array}\right)$ and $\pm\left(\begin{array}{ll}1 & 2 \\ 0 & 1\end{array}\right)$ (which map under $\rho$ to $S_{4} S_{3}$ and $S_{2} S_{3}$, respectively) generate the group

$$
\Lambda(2):=\left\{\left(\begin{array}{ll}
a & b \\
c & d
\end{array}\right) \in \mathrm{SL}_{2}(\mathbb{Z}): \begin{array}{l}
a \equiv d \equiv 1(\bmod 2) \\
b \equiv c \equiv 0(\bmod 2)
\end{array}\right\} .
$$

This is the so-called level-2 principal congruence subgroup of $\mathrm{SL}_{2}(\mathbb{Z})$. Hence by Lemma 3.32, the group $\Gamma$ contains

$$
\Xi:=\left\langle S_{2} S_{3}, S_{4} S_{3}\right\rangle=\rho(\Lambda(2)) .
$$

The point is that $\Lambda(2)$ is arithmetic, being defined in (3.35) by congruences. Then for any integer $\ell$ coprime to $2 k$, there is a matrix $\left(\begin{array}{cc}* & * \\ 2 k & \ell\end{array}\right)$ in $\Lambda(2)$. One can work out, with the same $\mathbf{v}_{0}$ and $\mathbf{w}_{0}$ as above, that

$$
\left\langle\mathbf{e}_{4}, \rho\left(\begin{array}{cc}
* & * \\
2 k & \ell
\end{array}\right) \cdot \mathbf{v}_{0}\right\rangle=32 k^{2}+24 k \ell+17 \ell^{2}+10 .
$$

For example, the choices $(2 k, \ell)=(4,-3),(2,-1),(4,-1)$, and $(6,-1)$ give curvatures $147,35,107$, and 243, respectively, visible up the left side of Figure 1, all 
tangent to the bounding circle (since $\Xi$ in 3.36 fixes $C_{1}$ ). Observe also that setting $\ell=1$ in (3.37) recovers (3.34). In this way, Sarnak Sar07] proved that the set $\mathscr{K}$ of represented numbers contains all primitive (meaning with $2 k$ and $\ell$ coprime) values of the shifted binary quadratic form in (3.37). Note that the quadratic form has discriminant $24^{2}-4 \cdot 32 \cdot 17=-1600$, and so (3.37) is definite, taking only positive values. The number of distinct primitive values of (3.37) up to $N$ was determined by Landau Lan08]: it is asymptotic to a constant times $N / \sqrt{\log N}$, thereby proving (3.26). A much more delicate and clever, but still "elementary" (no automorphic forms are harmed), argument goes into the proof of the Positive Density Conjecture (3.27), using an ensemble of such shifted binary quadratic forms. For Theorem A, one needs the theory of automorphic representations for the full Apollonian group, as hinted to at the end of 3.1 .6

We now leave the discussion of the Apollonian problem, returning to it again in $\$ 5$

\section{The thin Pythagorean PRoblem}

A Pythagorean triple $\mathbf{x}=(x, y, z)^{t}$ is a point on the cone

$$
Q(\mathbf{x})=0,
$$

where $Q$ is the "Pythagorean quadratic form"

$$
Q(\mathbf{x}):=x^{2}+y^{2}-z^{2} .
$$

Throughout we consider only integral triples, $\mathbf{x} \in \mathbb{Z}^{3}$, and assume that $x, y$, and $z$ are coprime; such a triple is called primitive. Elementary considerations then force the hypotenuse $z$ to be odd, and $x$ and $y$ to be of opposite parity; we assume henceforth that $x$ is odd and $y$ is even. The cone has a singularity at the origin, so we only consider its top half, assuming subsequently that the hypotenuse is positive, $z>0$.

Diophantus (and likely the Babylonians Pli] who preceded him by about as much as he precedes us) knew how to parametrize Pythagorean triples: Given $\mathbf{x}$, there is a pair $\mathbf{v}=(u, v)$ of coprime integers of opposite parity so that

$$
\left\{\begin{array}{l}
x=u^{2}-v^{2} \\
y=2 u v \\
z=u^{2}+v^{2} .
\end{array}\right.
$$

That the converse is true is elementary algebra: any such pair $\mathbf{v}$ inserted into (4.2) gives rise to a triple $\mathbf{x}$ satisfying (4.1). For example, it is easy to see that the triple

$$
\mathbf{x}_{0}=(3,4,5)^{t}
$$

corresponds to the pair

$$
\mathbf{v}_{0}=(2,1)^{t}
$$

4.1. Orbits and the spin representation. As in the Apollonian case, the Pythagorean form $Q$ has a special (determinant one) orthogonal group preserving it:

$$
\mathrm{SO}_{Q}:=\left\{g \in \mathrm{SL}_{3}: Q(g \cdot \mathbf{x})=Q(\mathbf{x})\right\} .
$$

And as before, this group is also better understood by passing to its spin cover. Since the Pythagorean form $Q$ has signature $(2,1)$, there is an accidental isomorphism between its spin group and $\mathrm{SL}_{2}(\mathbb{R})$, given explicitly as follows. 
Observe that $\mathrm{SL}_{2}$ acts on a pair $\mathbf{v}$ by left multiplication; via (4.2), this action then extends to a linear action on $\mathbf{x}$. In coordinates, it is an elementary computation that the action of $\left(\begin{array}{ll}a & b \\ c & d\end{array}\right)$ on $\mathbf{v}$ corresponds to left multiplication on $\mathbf{x}$ by

$$
\frac{1}{a d-b c}\left(\begin{array}{ccc}
\frac{1}{2}\left(a^{2}-b^{2}-c^{2}+d^{2}\right) & a c-b d & \frac{1}{2}\left(a^{2}-b^{2}+c^{2}-d^{2}\right) \\
a b-c d & b c+a d & a b+c d \\
\frac{1}{2}\left(a^{2}+b^{2}-c^{2}-d^{2}\right) & a c+b d & \frac{1}{2}\left(a^{2}+b^{2}+c^{2}+d^{2}\right)
\end{array}\right) .
$$

One can check directly from the definition (4.5) that (4.6) is an element of $\mathrm{SO}_{Q}$, in fact of the connected component $\mathrm{SO}_{Q}^{\circ}$ of the identity, and hence we have explicitly constructed the spin homomorphism

$$
\left.\rho: \mathrm{SL}_{2}(\mathbb{R}) \rightarrow \mathrm{SO}_{Q}(\mathbb{R}):\left(\begin{array}{ll}
a & b \\
c & d
\end{array}\right) \mapsto \underline{4.6}\right) .
$$

Given a Pythagorean triple $\mathbf{x}_{0}$, such as that in (4.3), the group $\Gamma:=\mathrm{SO}_{Q}^{\circ}(\mathbb{Z})$ of all integer matrices in $\mathrm{SO}_{Q}^{\circ}$ acts by left multiplication, giving the full orbit $\mathcal{O}=\Gamma \cdot \mathbf{x}_{0}$ of all Pythagorean triples (with our convention that $z>0, x$ is odd, and $y$ is even).

Via (4.2) again, this $\mathrm{SO}_{Q}$ action on $\mathbf{x}$ is equivalent to the $\mathrm{SL}_{2}$ action on $\mathbf{v}$. For a primitive $\mathbf{v} \in \mathbb{Z}^{2}$, both the integrality and primitivity are preserved by restricting the action to just the integral matrices $\mathrm{SL}_{2}(\mathbb{Z})$. Moreover, one should preserve the parity condition on $\mathbf{v}$ by restricting further to only the principal 2-congruence subgroup

$$
\Lambda(2)=\left\{\gamma \in \mathrm{SL}_{2}(\mathbb{Z}): \gamma \equiv I(\bmod 2)\right\}=\left\langle \pm\left(\begin{array}{ll}
1 & 2 \\
0 & 1
\end{array}\right), \pm\left(\begin{array}{ll}
1 & 0 \\
2 & 1
\end{array}\right)\right\rangle,
$$

which already appeared in 33.2 .2 . One can check directly that the image (4.6) of any $\gamma \in \Lambda(2)$ is an integral matrix, that is, in $\mathrm{SO}_{Q}(\mathbb{Z})$. For $\mathbf{v}_{0}$ corresponding to $\mathbf{x}_{0}$, the orbit $\tilde{\mathcal{O}}:=\tilde{\Gamma} \cdot \mathbf{v}_{0}$ under the full group $\tilde{\Gamma}:=\Lambda(2)$ consists of all coprime $(u, v)$ with $u$ even and $v$ odd.

Prompted by the Affine Sieve5 [BGS06, BGS10, SGS11] one may wish to study thin orbits $\mathcal{O}$ of Pythagorean triples. Here one replaces the full group $\mathrm{SO}_{Q}(\mathbb{Z})$ by some finitely generated subgroup $\Gamma$ of infinite index. Equivalently one can consider an orbit $\tilde{\mathcal{O}}$ of $\mathbf{v}_{0}$ under an infinite index subgroup $\tilde{\Gamma}$ of $\Lambda(2)$. We illustrate the general theory via the following concrete example.

We first give a sample $\tilde{\mathcal{O}}$ orbit: in comparison with the generators of $\Lambda(2)$, let $\tilde{\Gamma}$ be the group generated by the following two matrices

$$
\tilde{\Gamma}:=\left\langle \pm\left(\begin{array}{ll}
1 & 2 \\
0 & 1
\end{array}\right), \quad \pm\left(\begin{array}{ll}
1 & 0 \\
4 & 1
\end{array}\right)\right\rangle .
$$

This group clearly sits inside $\Lambda(2)$ but it is not immediately obvious whether it is of finite or infinite index; as we will see later, the index is infinite. Taking the base pair $\mathbf{v}_{0}$ in (4.4), we form the orbit

$$
\tilde{\mathcal{O}}:=\tilde{\Gamma} \cdot \mathbf{v}_{0} \cdot
$$

Correspondingly, we can take the base triple $\mathbf{x}_{0}$ in (4.3), and form the orbit

$$
\mathcal{O}:=\Gamma \cdot \mathbf{x}_{0}
$$

\footnotetext{
${ }^{5}$ We have insufficient room to survey this beautiful theory, for which the reader is directed to any number of excellent surveys; see e.g. SG12.
} 


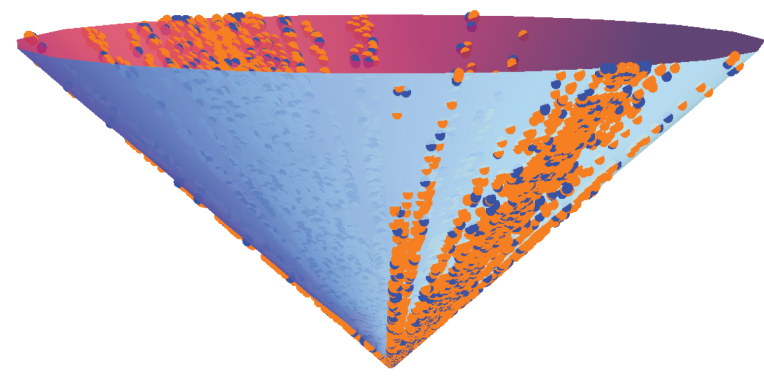

(a) View from the side.

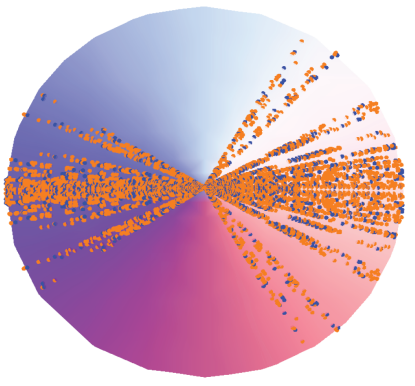

(b) View from below.

Figure 10. The thin Pythagorean orbit $\mathcal{O}$ in (4.9). Points are marked according to whether the hypotenuse is prime $(\mathbf{)}$ or composite (O).

of $\mathbf{x}_{0}$ under the group

$$
\Gamma:=\left\langle M_{1}, M_{2}\right\rangle
$$

where $M_{1}$ and $M_{2}$ are the images under $\rho$ of the matrices generating $\tilde{\Gamma}$. One can elementarily compute from (4.7) and (4.6) that

$$
M_{1}:=\left(\begin{array}{ccc}
-1 & -2 & -2 \\
2 & 1 & 2 \\
2 & 2 & 3
\end{array}\right), \quad M_{2}:=\left(\begin{array}{ccc}
-7 & 4 & 8 \\
-4 & 1 & 4 \\
-8 & 4 & 9
\end{array}\right) .
$$

Figure 10 illustrates this orbit $\mathcal{O}$. We can visually verify that the orbit looks thin, and in the next subsection we confirm this rigorously.

4.2. The orbit is thin. The group $\mathrm{SL}_{2}(\mathbb{R})$ also acts on the hyperbolic upper halfplane

$$
\mathbb{H}:=\{z=x+i y: x \in \mathbb{R}, y>0\}
$$

by fractional linear transformations,

$$
\left(\begin{array}{cc}
a & b \\
c & d
\end{array}\right): z \mapsto \frac{a z+b}{c z+d}
$$

The action of our group $\tilde{\Gamma}$ in (4.7) on $\mathbb{H}$ has a fundamental domain (the definition is similar to (3.10) ) given by

$$
\{z \in \mathbb{H}:|\mathfrak{R e}(z)|<1,|z-1 / 4|>1 / 4,|z+1 / 4|>1 / 4\},
$$

where the distances above are Euclidean; see Figure 11a. The hyperbolic measure is $y^{-2} d x d y$, and hence this region again has infinite hyperbolic area. Equivalently, the index of $\tilde{\Gamma}$ in $\Lambda(2)$ is infinite (it is well known that $\Lambda(2)$ has finite co-area), as claimed.

Any orbit of a fixed basepoint in $\mathbb{H}$ under $\tilde{\Gamma}$ has some limit set $\mathscr{C}=\mathscr{C}(\tilde{\Gamma})$ in the boundary $\partial \mathbb{H}$. A piece of this Cantor-like set can already be seen in Figure 11]. But to see it fully, we show in Figure 11b the same $\tilde{\Gamma}$-orbit in the disk model

$$
\mathbb{D}=\{z \in \mathbb{C}:|z|<1\},
$$




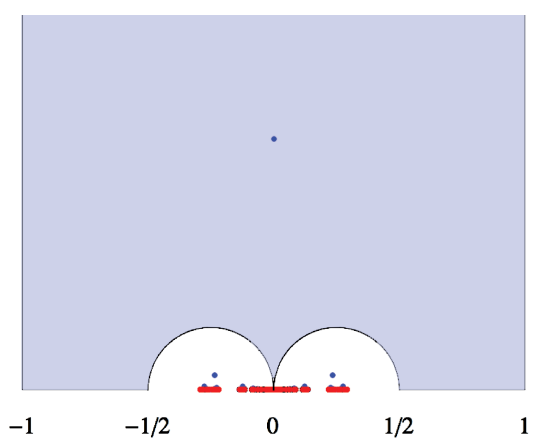

(a) Upper half plane model.

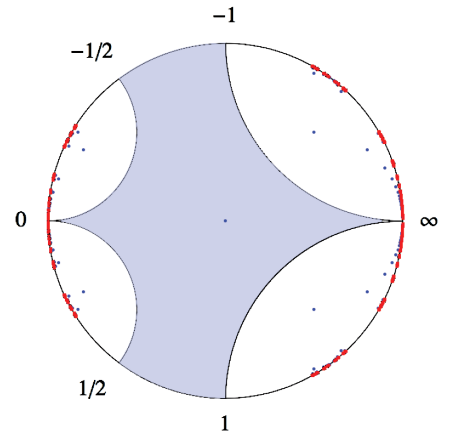

(b) Disk model.

Figure 11. The orbit of $i \in \mathbb{H}$ under $\tilde{\Gamma}$.

by composing the action of $\tilde{\Gamma}$ with the map

$$
\mathbb{H} \rightarrow \mathbb{D}: z \mapsto \frac{z-i}{z+i}
$$

(which encodes the observation that points in the upper half-plane are closer to $i$ than they are to $-i$ ). In the disk model, one more clearly sees the limit set as the set of "directions" in which the orbit $\mathcal{O}$ can grow-juxtapose Figure 10b on Figure 11]. This limit set $\mathscr{C}$ has some Hausdorff dimension $\delta=\delta(\tilde{\Gamma}) \in[0,1]$; one can estimate

$$
\delta \approx 0.59 \cdots .
$$

This dimension (also called the "critical exponent of $\Gamma$ ") is again an important geometric invariant, measuring the "thinness" of $\Gamma$, as illustrated in the following counting statement Kon07, Kon09, KO12. Let $\|\mathbf{x}\|$ be the Euclidean norm. There is some $c>0$ so that

$$
\#\{\mathbf{x} \in \mathcal{O}:\|\mathbf{x}\|<N\} \sim c N^{\delta}, \quad \text { as } N \rightarrow \infty .
$$

Once again, (4.14) should be compared with the orbit of $\mathbf{x}_{0}$ under the full ambient group, $\mathrm{SO}_{Q}(\mathbb{Z})$. Elementary methods show that

$$
\#\left\{\mathbf{x} \in \mathrm{SO}_{Q}(\mathbb{Z}) \cdot \mathbf{x}_{0}:\|\mathbf{x}\|<N\right\} \sim c N .
$$

So in passing from the full orbit to $\mathcal{O}$, the asymptotic drops from $N$ to $N^{\delta}$, with $\delta<1$. Thus the orbit $\mathcal{O}$ is thin.

The fact that $\rho$ is a quadratic map in the entries (see (4.6)) implies that the count (4.14) on triples $\mathbf{x} \in \mathcal{O}$ is equivalent to the following asymptotic for the pairs $\mathbf{v} \in \tilde{\mathcal{O}}:$

$$
\#\{\mathbf{v} \in \tilde{\mathcal{O}}:\|\mathbf{v}\|<N\} \sim c^{\prime} \cdot N^{2 \delta},
$$

as $N \rightarrow \infty$. Note that the power of $N$ is now $2 \delta$. It can also be seen immediately from (4.1) and (4.2) that

$$
\|\mathbf{x}\|=\sqrt{x^{2}+y^{2}+z^{2}}=\sqrt{2} z=\sqrt{2}\left(u^{2}+v^{2}\right)=\sqrt{2}\|\mathbf{v}\|^{2} .
$$

(Geometrically, the cone (4.1) intersects the sphere of radius $N$ at a circle of radius $N / \sqrt{2}$.) Observe that (4.14) looks like the Apollonian asymptotic (3.5), while (4.15) 
is more similar to Hensley's estimate (2.17) in Zaremba's problem. This is just a consequence of choosing between working in the orthogonal group or its spin cover.

4.3. Diophantine problems. One can now pose a variety of Diophantine questions about the values of various functions on such thin orbits. Given an orbit $\mathcal{O}=\Gamma \cdot \mathbf{x}_{0}$ and a function $f: \mathcal{O} \rightarrow \mathbb{Z}$, call

$$
\mathscr{P}:=f(\mathcal{O}) \subset \mathbb{Z}
$$

the set of represented numbers. That is, $n$ is represented by the pair $(\mathcal{O}, f)$ if there is some $\gamma \in \Gamma$ so that $n=f\left(\gamma \cdot \mathbf{x}_{0}\right)$. And as before, we say $n$ is admissible if $n \in \mathscr{P}(\bmod q)$ for all $q$. For example, if $f$ is the "hypotenuse" function, $f(\mathbf{x})=z$, one can ask whether $(\mathcal{O}, f)$ represents infinitely many admissible primes. Evidence to the affirmative is illustrated in Figure 10, where a triple is highlighted if its hypotenuse is prime. Unfortunately this problem on thin orbit:6 seems out of reach of current technology.

But for a restricted class $\mathcal{F}$ of functions $f$, and orbits $\mathcal{O}$ which are "not too thin", recent progress has been made toward the local-global problem in $\mathscr{P}$. Let $\mathcal{F}$ be the set of functions $f$ which are a linear, not on the triples $\mathbf{x}$, but on the corresponding pairs v. For example, it is not particularly well known that in a Pythagorean triple, the sum of the hypotenuse $z$ and the even side $y$ is always a perfect square. This follows immediately from the parametrization (4.2); in particular, $y+z=(u+v)^{2}$. So the function

$$
f(\mathbf{x})=\sqrt{y+z}=u+v
$$

is integer valued on $\mathcal{O}$ and linear 7 in $\mathbf{v}$.

Another way of saying this is to pass to the corresponding orbit $\tilde{\mathcal{O}}=\tilde{\Gamma} \cdot \mathbf{v}_{0}$. Any such linear function on $\mathbf{v}$ is of the form

$$
f(\mathbf{v})=\left\langle\mathbf{w}_{0}, \mathbf{v}\right\rangle,
$$

for some fixed $\mathbf{w}_{0} \in \mathbb{Z}^{2}$. In the example (4.18), take $\mathbf{w}_{0}=(1,1)^{t}$. Then $\mathcal{F}$ consists of all functions on $\mathcal{O}$ which, pulled back to $\tilde{\mathcal{O}}$, are of the form (4.19).

Theorem P (Bourgain and Kontorovich, 2010 [BK10]). Fix any such linear $f \in \mathcal{F}$ and Pythagorean triple $\mathbf{x}_{0}$. There is some $\delta_{0}<1$ (the value $\delta_{0}=0.99995$ suffices) so that if the orbit $\mathcal{O}=\Gamma \cdot \mathbf{x}_{0}$ is not too thin, meaning the exponent $\delta$ of $\Gamma$ satisfies

$$
\delta>\delta_{0}
$$

then almost every admissible number is represented in $\mathscr{P}=f(\mathcal{O})$.

We are finally in position to relate this Pythagorean problem to the Apollonian and Zaremba's. Indeed, passing to the corresponding orbit $\tilde{\mathcal{O}}=\tilde{\Gamma} \cdot \mathbf{v}_{0}$ and fixing the function $f(\mathbf{v})=\left\langle\mathbf{w}_{0}, \mathbf{v}\right\rangle$, we have that $n$ is represented if there is a $\gamma \in \tilde{\Gamma}$ so that

$$
n=\left\langle\mathbf{w}_{0}, \gamma \cdot \mathbf{v}_{0}\right\rangle
$$

\footnotetext{
${ }^{6}$ For the full orbit of all Pythagorean triples, infinitely many hypotenuses are prime. This follows from (4.2) that $z=u^{2}+v^{2}$ and Fermat's theorem that all primes $\equiv 1(\bmod 4)$ are sums of two squares.

${ }^{7}$ Really we want the values of $|u+v|$, which within the positive integers are the union of the values of $u+v$ and $-u-v$. Alternatively, we can assume that $-I \in \tilde{\Gamma}$, as is the case for (4.7).
} 
That is,

$$
\mathscr{P}=\left\langle\mathbf{w}_{0}, \tilde{\Gamma} \cdot \mathbf{v}_{0}\right\rangle,
$$

which is of the same form as (2.29) and (3.28). The condition of admissibility is analyzed again given the generators of $\tilde{\Gamma}$ by strong approximation, Goursat's Lemma, and finite group theory, as in 2.2 .

Note that in light of the asymptotic counting formula (4.15), the minimal dimension $\delta_{0}$ in (4.20) cannot go below $1 / 2$ : the numbers in $\mathscr{P}$ up to $N$ (counted with multiplicity) have cardinality roughly $N^{2 \delta}$, so if $\delta$ is less than $1 / 2$, then certainly a local-global principle fails miserably. (Such a phenomenon appeared already in the context of Hensley's conjecture (2.15) in Zaremba's problem.)

\section{The CIRCle METHOD: TOOLS AND PROOFS}

We briefly review the previous three sections, unifying the (re)formulations of the problems. The Apollonian, Pythagorean, and Zaremba theorems will henceforth be referred to as Theorem $X$, where

$$
X=\mathrm{AlP} \text {, or Z, }
$$

respectively. Theorem $X$ concerns the set $\mathscr{S}$ of numbers of the form

$$
\mathscr{S}=\left\langle\mathbf{w}_{0}, \Gamma \cdot \mathbf{v}_{0}\right\rangle \text {. }
$$

Here

$$
\begin{array}{r}
\mathscr{S}= \begin{cases}\mathscr{K}=\text { the set of curvatures }(3.28) & \text { if } X=\mathrm{A}, \\
\mathscr{P}=\text { the set of square-roots of sums of } & \text { if } X=\mathrm{P} \\
\text { hypotenuses and even sides }(4.22),(4.18) & \text { if } X=\mathrm{Z},\end{cases} \\
\Gamma= \begin{cases}\text { the Apollonian group } \Gamma & \text { if } X=\mathrm{A}, \\
\text { an infinite index subgroup } \tilde{\Gamma}<\Lambda(2) & \text { if } X=\mathrm{P} \\
\text { the semigroup } \Gamma_{\mathcal{A}} & \text { if } X=\mathrm{Z}\end{cases} \\
\mathbf{v}_{0}= \begin{cases}\text { the root quadruple } & \text { if } X=\overline{\mathrm{A}}, \\
\text { any coprime pair of opposite parity } & \text { if } X=\mathrm{P} \\
(0,1)^{t} & \text { if } X=\sqrt{\mathrm{Z}},\end{cases}
\end{array}
$$

and

$$
\mathbf{w}_{0}= \begin{cases}\text { a standard basis vector } \mathbf{e}_{j} & \text { if } X=\mathrm{A} \\ \text { any fixed pair } & \text { if } X=\mathrm{P} \\ (0,1)^{t} & \text { if } X=\mathrm{Z}\end{cases}
$$

But now we can forget the individual problems and just focus on the general setting (5.1); one need not keep the above taxonomy in one's head throughout.

To study the local-global problem for $\mathscr{S}$, we introduce the representation function

$$
\mathcal{R}_{N}(n):=\sum_{\gamma \in \Omega_{N}} \mathbf{1}_{\left\{n=\left\langle\mathbf{w}_{0}, \gamma \cdot \mathbf{v}_{0}\right\rangle\right\}}
$$

Here $N$ is a growing parameter and $\Omega_{N}$ is a certain subset of the radius $N$ ball in $\Gamma$,

$$
\Omega_{N} \subset\{\gamma \in \Gamma:\|\gamma\|<N\}
$$


which we will describe in more detail later. For now, one can just think of $\Omega_{N}$ as the whole radius $N$ ball. To get our bearings, let us recall roughly 8 the size of $\Omega_{N}$ :

$$
\#\{\gamma \in \Gamma:\|\gamma\|<N\} \asymp \begin{cases}N^{\delta}, & \text { if } X=\mathrm{A} \text {, see (3.5), } \\ N^{2 \delta}, & \text { if } X=\mathrm{P} \text {, see (4.15), } \\ N^{2 \delta_{\mathcal{A}}}, & \text { if } X=\mathrm{Z} \text {, see (2.17). }\end{cases}
$$

We can write this uniformly by introducing the parameter $\alpha$, defined by

$$
\alpha:= \begin{cases}\delta, \text { the dimension of an Apollonian packing } & \text { if } X=\mathrm{A} \text {, see (3.4), } \\ 2 \delta, \text { where } \delta \text { is the dimension of } \mathscr{C}(\tilde{\Gamma}), & \text { if } X=\mathrm{P} \text {, see (4.13) } \\ 2 \delta_{\mathcal{A}}, \text { where } \delta_{\mathcal{A}} \text { is the dimension of } \mathscr{C}_{\mathcal{A}} & \text { if } X=\mathrm{Z}, \text { see (2.10). }\end{cases}
$$

In each case $\alpha$ satisfies

$$
1<\alpha<2 \text {. }
$$

Then the cardinality of such a ball $\Omega_{N}$ is roughly

$$
\left|\Omega_{N}\right| \asymp N^{\alpha} \text {. }
$$

Returning to (5.2), we see by construction that $\mathcal{R}_{N}$ is nonnegative. Moreover observe that

$$
\text { if } \mathcal{R}_{N}(n)>0 \text {, then certainly } n \text { is represented in } \mathscr{S} \text {. }
$$

Also record that

$$
\mathcal{R}_{N} \text { is supported on } n \text { of size }|n| \ll N \text {. }
$$

Recalling the notation $e(x)=e^{2 \pi i x}$, the Fourier transform

$$
\begin{aligned}
\mathcal{S}_{N}(\theta) & :=\widehat{\mathcal{R}_{N}}(\theta)=\sum_{n \in \mathbb{Z}} \mathcal{R}_{N}(n) e(n \theta) \\
& =\sum_{\gamma \in \Omega_{N}} e\left(\theta\left\langle\mathbf{w}_{0}, \gamma \cdot \mathbf{v}_{0}\right\rangle\right)
\end{aligned}
$$

is a wildly oscillating exponential sum on the circle $\mathbb{R} / \mathbb{Z}=[0,1)$, whose graph looks something like Figure 12. One recovers $\mathcal{R}_{N}$ through elementary Fourier inversion,

$$
\mathcal{R}_{N}(n)=\int_{\mathbb{R} / \mathbb{Z}} \mathcal{S}_{N}(\theta) e(-n \theta) d \theta,
$$

but without further ingredients, one is going around in circles (no pun intended).

Hardy and Littlewood had the idea that the bulk of the integral (5.8) could be captured just by integrating over frequencies $\theta$ that are very close to rational numbers $a / q,(a, q)=1$, with very small denominators $q$; some of these intervals are shaded in Figure 12. These are now called the major arcs $\mathfrak{M}$; the name refers not to their total length (they comprise a tiny fraction of the circle $\mathbb{R} / \mathbb{Z}$ ) but to the fact that they are supposed to account for a preponderance of $\mathcal{R}_{N}(n)$. Accordingly, we decompose (5.8) as

$$
\mathcal{R}_{N}(n)=\mathcal{M}_{N}(n)+\mathcal{E}_{N}(n),
$$

where the major arc contribution

$$
\mathcal{M}_{N}(n):=\int_{\mathfrak{M}} \mathcal{S}_{N}(\theta) e(-n \theta) d \theta
$$

\footnotetext{
${ }^{8}$ Technically the quoted results are about counting in the corresponding orbits $\mathcal{O}$ and not in the groups $\Gamma$, but the order of magnitude is the same for both.
} 


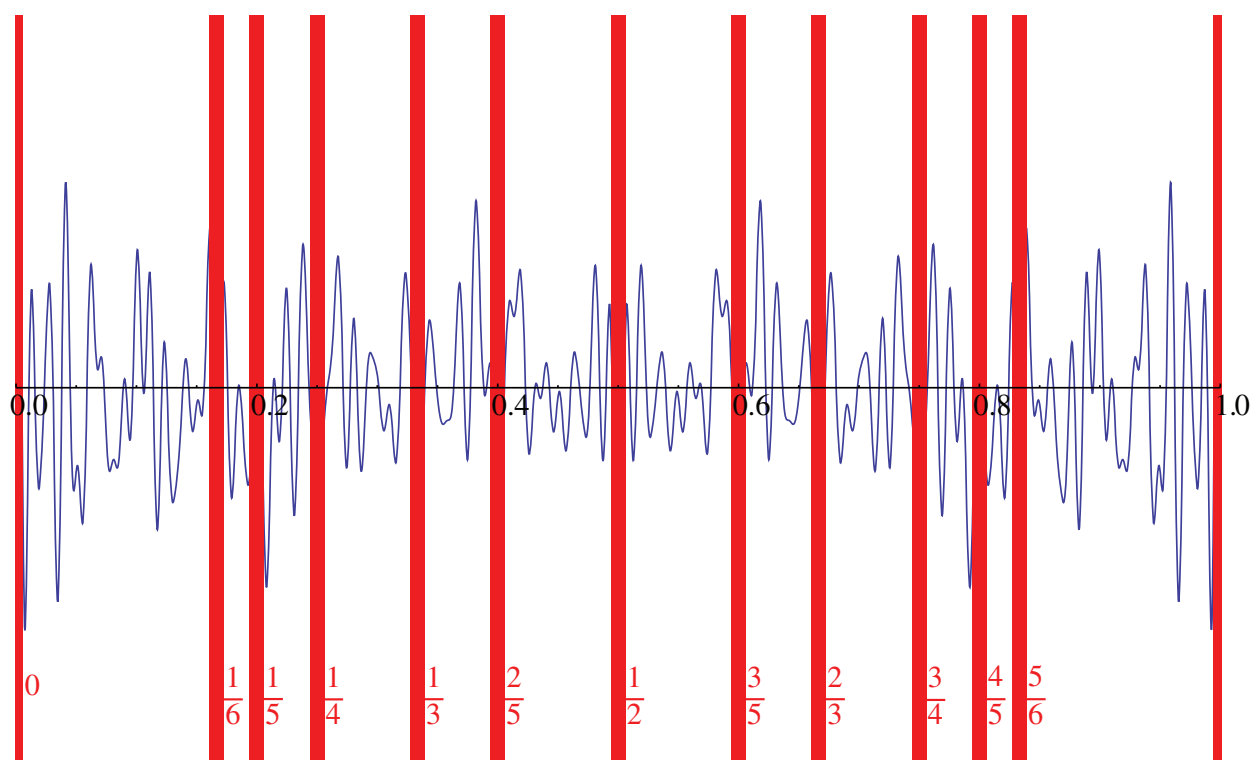

FiguRE 12. The real part of an exponential sum of the form (5.7).

is supposed to give the "main" term, and

$$
\mathcal{E}_{N}(n):=\int_{\mathfrak{m}} \mathcal{S}_{N}(\theta) e(-n \theta) d \theta
$$

should be the "error". Here $\mathfrak{m}:=[0,1) \backslash \mathfrak{M}$ are the complemenary so-called minor $\operatorname{arcs.~If~} \mathcal{M}_{N}(n)$ is positive and bigger than $\left|\mathcal{E}_{N}(n)\right|$, then certainly

$$
\mathcal{R}_{N}(n) \geq \mathcal{M}_{N}(n)-\left|\mathcal{E}_{N}(n)\right|>0,
$$

so again, $n$ is represented. In practice, one typically tries to prove an asymptotic formula (or at least a lower bound) for $\mathcal{M}_{N}$, and then give an upper bound for $\left|\mathcal{E}_{N}\right|$.

The reason for this decomposition is that exponential sums such as $\mathcal{S}_{N}$ should be mostly supported on $\mathfrak{M}$, having their biggest peaks and valleys at (or very near) these frequencies (some of this phenomenon is visible in Figure 12). Indeed, the value $\theta=0$ is as big as $\mathcal{S}_{N}$ will ever get,

$$
\left|\mathcal{S}_{N}(\theta)\right| \leq \mathcal{S}_{N}(0)=\left|\Omega_{N}\right|
$$

which follows trivially (and is thus called the trivial bound) from the triangle inequality: every summand in (5.7) is a complex number of absolute value 1. Also for other $\theta \in \mathfrak{M}, \theta \approx a / q$, the summands should all point in a limited number of directions, colluding to give a large contribution to $\mathcal{S}_{N}$. As we will see later, at these frequencies, one is in a sense measuring the distribution of $\mathscr{S}$ (or equivalently $\Omega_{N}$ ) along certain arithmetic progressions. This strategy of coaxing out the (conjectural) main term for $\mathcal{R}_{N}$ works in surprisingly great generality, but can also give false predictions (even for the Prime Number Theorem, see e.g. Gra95]). 
Having made this decomposition, we should determine what we expect for the main term. From (5.7), we have that

$$
\sum_{n} \mathcal{R}_{N}(n)=\mathcal{S}_{N}(0)=\left|\Omega_{N}\right|
$$

so recalling the support (5.2) of $\mathcal{R}_{N}$, one might expect that an admissible number of size about $n \asymp N$ is represented roughly $\left|\Omega_{N}\right| / N$ times. In particular, since every admissible number is expected to be represented, one would like to show, say, for $N / 2 \leq n<N$, that

$$
\mathcal{M}_{N}(n) \gg \mathfrak{S}(n) \frac{\left|\Omega_{N}\right|}{N} .
$$

Here $\mathfrak{S}(n) \geq 0$ is a certain product of local densities called the singular series; it alone is responsible for the notion of admissibility, vanishing on nonadmissible $n$. For admissible $n$, it typically does not fluctuate too much; crudely, one can show in many contexts the lower bound $\gg N^{-\varepsilon}$ for any $\varepsilon>0$. For ease of exposition, let us just pretend for now that every $n$ is admissible and remove the role of the singular series, allowing ourselves to assume that

$$
\mathfrak{S}(n)=1 .
$$

Observe also that, in light of the cardinality (5.4) of $\left|\Omega_{N}\right|$ and with exponent $\alpha$ ranging in (5.3), the lower bound in (5.13) is of the order $N^{\alpha-1}$, with $\alpha>1$. That is, there should be quite a lot of representations of an admissible $n \asymp N$ large, giving further indication that every sufficiently large admissible number may be represented.

One is then left with the problem of estimating away the remainder term $\mathcal{E}_{N}$, and this is why (as Peter Sarnak likes to say) the circle method is a "method" and not a "theorem": establishing such estimates is much more of an art than a science. The Hardy-Littlewood procedure suggests somehow exploiting the fact that on the minor arc frequencies, $\theta \in \mathfrak{m}$, the exponential sum $\mathcal{S}_{N}$ in (5.7) should itself already be quite small, being a sum of canceling phases. If one could indeed prove at the level of individual $n$ an upper bound for the error term $\mathcal{E}_{N}$, which is asymptotically smaller than the lower bound (5.13) for $\mathcal{M}_{N}$, then one would immediately conclude the full local-global conjecture that every sufficiently large admissible $n$ is represented. Unfortunately, at present we do not know how to give such strong upper bounds on the minor arcs.

Instead, we settle for an "almost" local-global statement, by proving a sharp bound not for individual $n$, but for $n$ in an average sense, as follows. Parseval's theorem states that the $L^{2}$-norm of a function is equal to that of its Fourier transform; that is, the Fourier transform is a unitary operator on these Hilbert spaces. Using the definition (5.10), Parseval's theorem then gives

$$
\sum_{n}\left|\mathcal{E}_{N}(n)\right|^{2}=\int_{\mathfrak{m}}\left|\mathcal{S}_{N}(\theta)\right|^{2} d \theta .
$$

Inserting our trivial bound (5.12) for $\mathcal{S}_{N}$ into the above yields a trivial bound for (5.15) of

$$
\int_{\mathfrak{m}}\left|\mathcal{S}_{N}(\theta)\right|^{2} d \theta \leq\left|\Omega_{N}\right|^{2}
$$


We claim that it suffices for our applications to establish a bound of the form

$$
\int_{\mathfrak{m}}\left|\mathcal{S}_{N}(\theta)\right|^{2} d \theta=o\left(\frac{\left|\Omega_{N}\right|^{2}}{N}\right) .
$$

That is, the above saves a little more than $\sqrt{N}$ on average over $\mathfrak{m}$ off of each term $\mathcal{S}_{N}$ relative to the trivial bound (5.16). We first explain why this suffices.

5.1. Proof of Theorem $X$, assuming (15.13) and (5.17). Let $\mathfrak{E}(N)$ be the set of exceptional $n$ (those that are admissible but not represented) in the range $N / 2 \leq$ $n<N$. Recalling the sufficient condition (5.11) for representation, the number of exceptions is bounded by

$$
\# \mathfrak{E}(N) \leq \sum_{\substack{N / 2<|n|<N \\ n \text { is admissible }}} \mathbf{1}_{\left\{\left|\mathcal{E}_{N}(n)\right| \geq \mathcal{M}_{N}(n)\right\}}
$$

For admissible $n$, we have the supposed major arc lower bound (5.13) and recall our simplifying assumption (5.14) to ignore the singular series; thus

$$
\# \mathfrak{E}(N) \leq \sum_{n} \mathbf{1}_{\left\{\left|\mathcal{E}_{N}(n)\right| \gg\left|\Omega_{N}\right| / N\right\}}
$$

Here is a pleasant (standard) trick: For those $n$ contributing a 1 rather than 0 to (5.18), we have

$$
1 \ll \frac{\left|\mathcal{E}_{N}(n)\right|}{\left|\Omega_{N}\right| / N},
$$

both sides of which may be squared. Hence (5.18) implies that

$$
\# \mathfrak{E}(N) \ll \frac{N^{2}}{\left|\Omega_{N}\right|^{2}} \cdot \sum_{n}\left|\mathcal{E}_{N}(n)\right|^{2} .
$$

Now we apply Parseval's theorem (5.15) and the supposed minor arcs bound (5.17). This gives

$$
\# \mathfrak{E}(N)=o\left(\frac{N^{2}}{\left|\Omega_{N}\right|^{2}} \cdot \frac{\left|\Omega_{N}\right|^{2}}{N}\right)=o(N),
$$

and thus $100 \%$ of the admissible numbers in the range $[N / 2, N)$ are represented. Combining such dyadic intervals, we conclude that almost every admissible number is represented.

Now "all" that is left is to establish the major arcs bound (5.13) and the error bound (5.17). In the next two subsections, we focus individually on the tools needed to prove these claims.

5.2. The major arcs. Recall that $\mathcal{M}_{N}$ in (5.9) is an integral over the major arcs $\theta \in \mathfrak{M}$; here $\theta$ is very close to a fraction $a / q$, with $q$ "small" (the meaning of which is explained below). Also let us pretend for now that $\Omega_{N}$ is just the whole $\Gamma$-ball,

$$
\Omega_{N}=\{\gamma \in \Gamma:\|\gamma\|<N\} .
$$

We begin by trying to evaluate (5.7) at $\theta=a / q$ :

$$
\mathcal{S}_{N}\left(\frac{a}{q}\right)=\sum_{\substack{\gamma \in \Gamma \\\|\gamma\|<N}} e\left(\frac{a}{q}\left\langle\mathbf{w}_{0}, \gamma \cdot \mathbf{v}_{0}\right\rangle\right) .
$$

An important observation in the above is that the summation may be grouped according to the residue class $\bmod q$ of the integer $\left\langle\mathbf{w}_{0}, \gamma \cdot \mathbf{v}_{0}\right\rangle$. Or what is essentially 


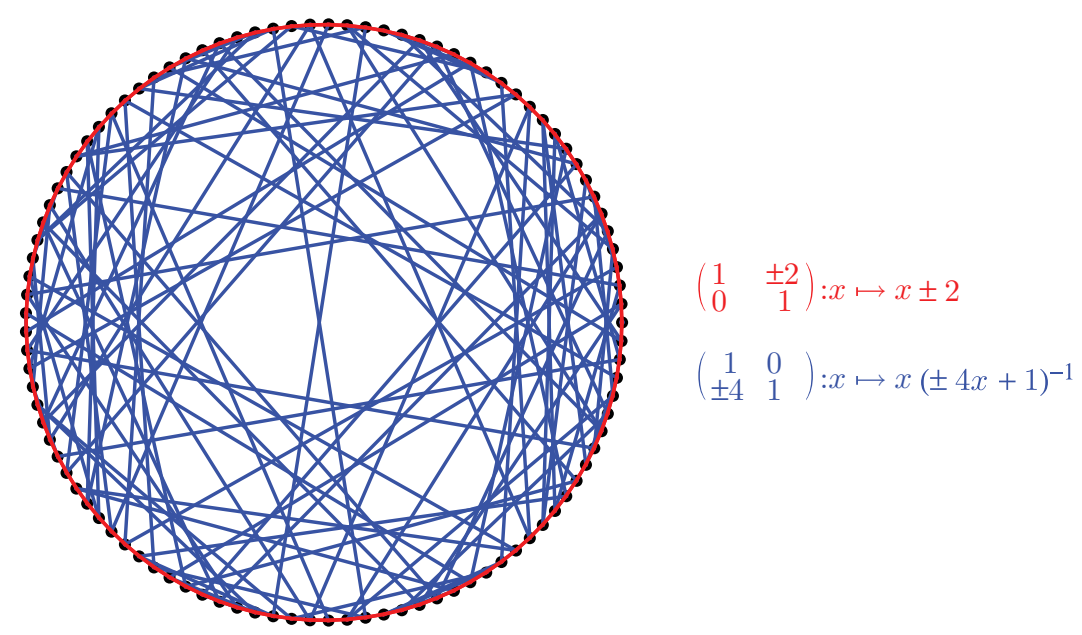

FIGURE 13. An expander; shown with $q=101$.

the same, we can decompose the sum according to the the residue class of $\gamma(\bmod q)$. To this end, let $\Gamma_{q}=\Gamma(\bmod q)$ be the set of such residue classes (which we have already studied in the context of admissibility and strong approximation). Then we split the sum as

$$
\mathcal{S}_{N}\left(\frac{a}{q}\right)=\left|\Omega_{N}\right| \sum_{\gamma_{0} \in \Gamma_{q}} e\left(\frac{a}{q}\left\langle\mathbf{v}_{0} \cdot \gamma_{0}, \mathbf{w}_{0}\right\rangle\right) \cdot\left[\frac{1}{\left|\Omega_{N}\right|} \sum_{\substack{\gamma \in \Gamma \\\|\gamma\|<N}} \mathbf{1}_{\left\{\gamma \equiv \gamma_{0}(\bmod q)\right\}}\right],
$$

where we have artificially multiplied and divided by the cardinality of $\Omega_{N}$. Now for $\gamma_{0}$ fixed, the bracketed term is then measuring the "probability" that $\gamma \equiv$ $\gamma_{0}(\bmod q)$. As one may suspect, our groups do not have particular preferences for certain residue classes over others; that is, this probability becomes equidistributed as $N$ grows, with $q$ also allowed to grow, but at a much slower rate. (In fact, this is exactly what we mean by the denominator $q$ being "small" - relative to $N$-in the major arcs $\mathfrak{M}$.) To explain how this happens, we briefly discuss the crucial notion of an expander.

Rather than going into the general theory (for which we refer the reader to the beautiful survey [Lub12]; see also [Sar04]), we content ourselves with but one illustrative example of expansion. Figure 13 shows the following graph. For $q=101$, say, take the vertices to be the elements of $\mathbb{Z} / q \mathbb{Z}$, organized around the unit circle by placing $x \in \mathbb{Z} / q \mathbb{Z}$ at $e(x / q)$. For the edges, connect each

$$
x \text { to } x \pm 2, \quad \text { and also to } x( \pm 4 x+1)^{-1} \text {, }
$$

when inversion $(\bmod q)$ is possible. This is nothing more than the fractional linear action (see (4.12) ) of the generating matrices in (4.7) (and their inverses) on $\mathbb{Z} / q \mathbb{Z}$. We first claim that our graph on $q$ vertices is "sparse". Indeed, the complete graph (connecting any vertex to any other) has on the order of $q^{2}$ edges, whereas our graph has only on the order of $q$ edges (since (5.21) implies that any vertex is connected to at most four others). So we have square-root the total number of possible edges, and our graph is indeed quite sparse. 
Despite having few edges, it is a fact that this graph is nevertheless highly connected, in the sense that a random walk on it is rapidly mixing. Moreover, this rate of mixing, properly normalized, is independent of the choice of $q$ above. That is, by varying $q$, we in fact have a whole family of such sparse but highly connected graphs, and with a uniform mixing rate; this is exactly what characterizes an expander.

Proofs of expansion use, among other things, tools from additive combinatorics, in particular, the so-called sum-product BKT04,Bou08 and triple-product [Hel08,BGT11,PS10] estimates, and quite a lot of other work which we will not survey; see e.g. [SX91, Gam02,BG08, BGS10,Var10, BV11,SGV11]. Once one proves uniform expansion for such finite graphs, the statements must be converted into the Archimedean form needed for the bracketed term in (5.20). To handle such counting statements, one uses

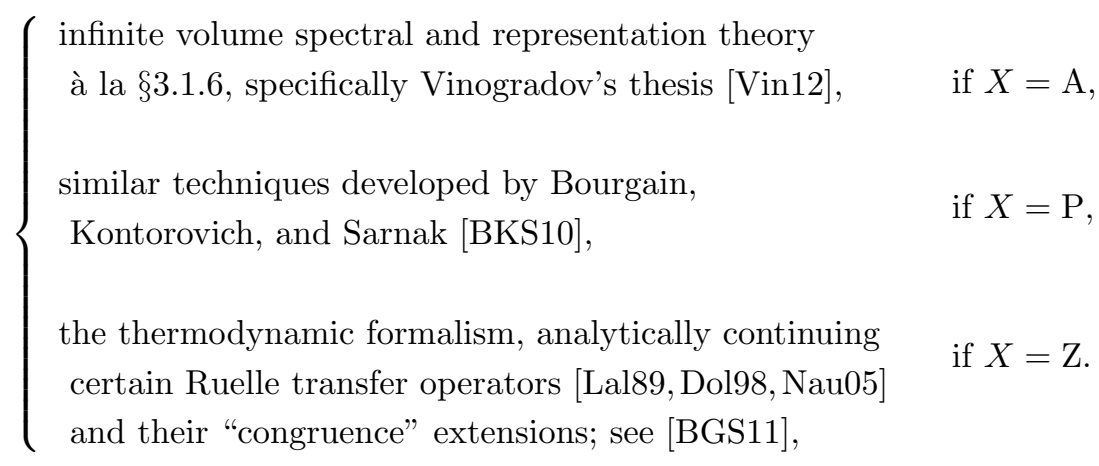

Without going into details, the upshot is that, up to acceptable errors, the bracketed term in (5.20) is just $1 /\left|\Gamma_{q}\right|$, confirming the desired equidistribution. Inserting this estimation into $\mathcal{M}_{N}$ in (5.9), one uses these techniques and some more standard circle method analysis to eventually conclude (5.13).

5.3. The minor arcs. We use different strategies to prove the minor arcs bound (5.17) for the Pythagorean and Zaremba settings $X=\mathrm{P}$ or Z, versus the Apollonian setting $X=\mathrm{A}$, so we present them individually. As the details quickly become quite technical, we will only scratch the surface, inviting the interested reader to study the original manuscripts BK10, BK11, BK12]. Hopefully the short sketches below give some indication for the flavor of the arguments involved.

5.3.1. Pythagorean and Zaremba settings. To handle the minor arcs here, we make the observation that the ensemble $\Omega_{N}$ in the definition of $\mathcal{S}_{N}$ from (5.7) need not be an exact $\Gamma$-ball as in (5.19), but can be replaced by, say, a product of two such. That is, the definition of $\mathcal{S}_{N}$ can be changed to

$$
\mathcal{S}_{N}(\theta):=\sum_{\substack{\gamma_{1} \in \Gamma \\\left\|\gamma_{1}\right\|<\sqrt{N}}} \sum_{\substack{\gamma_{2} \in \Gamma \\\left\|\gamma_{2}\right\|<\sqrt{N}}} e\left(\theta\left\langle\mathbf{v}_{0} \gamma_{1} \gamma_{2}, \mathbf{w}_{0}\right\rangle\right),
$$

without irreparably damaging the major arcs analysis. This new sum encodes much more of the (semi)group structure of $\Gamma$, while preserving the "nonvanishing implies represented" property (5.5), where $\mathcal{R}_{N}$ is redefined by Fourier inversion (5.8). (In reality, we use even more complicated exponential sums.) The advantage of (5.22) is that we can now exploit this structure à la Vinogradov's method [Vin37] for estimating bilinear forms: one can think of (5.22) as the sum of all entries in 
the matrix indexed by $\gamma_{1}$ and $\gamma_{2}$ with entries $e\left(\theta\left\langle\mathbf{v}_{0} \gamma_{1} \gamma_{2}, \omega_{0}\right\rangle\right)$. Just one standard maneuver in estimating bilinear forms is the following.

Apply the Cauchy-Schwarz inequality to (5.22) in the $\gamma_{1}$ variable:

$$
\left|\mathcal{S}_{N}(\theta)\right| \leq\left(\sum_{\substack{\gamma_{1} \in \Gamma \\\left\|\gamma_{1}\right\|<\sqrt{N}}} 1\right)^{1 / 2}\left(\sum_{\substack{\gamma_{1} \in \mathrm{SL}_{2}(\mathbb{Z}) \\\left\|\gamma_{1}\right\|<\sqrt{N}}}\left|\sum_{\substack{\gamma_{2} \in \Gamma \\\left\|\gamma_{2}\right\|<\sqrt{N}}} e\left(\theta\left\langle\mathbf{v}_{0} \gamma_{1} \gamma_{2}, \mathbf{w}_{0}\right\rangle\right)\right|^{2}\right)^{1 / 2} .
$$

Notice in the second appearance of a $\gamma_{1}$ sum, we have replaced the thin and mysterious group $\Gamma$ (or semigroup $\Gamma_{A}$ ) by the full ambient group $\mathrm{SL}_{2}(\mathbb{Z})$. On one hand, this allows us to now use more classical tools to get the requisite cancellation (5.17) in the minor arcs integral. On the other hand, this type of perturbation argument only succeeds when $\delta$ is near 1, explaining the dimension restrictions (2.23) and (4.20).

5.3.2. The Apollonian case. The above strategy fails for the Apollonian problem, because the Hausdorff dimension (3.4) is a fixed invariant which refuses to be adjusted to suit our needs. Instead, we recall that the Apollonian group $\Gamma$ contains the special (arithmetic) subgroup $\Xi$ from (3.36). Then, like (5.22), we change the definition of the exponential sum to something of the (again, bilinear) form

$$
\mathcal{S}_{N}(\theta):=\sum_{\substack{\xi \in \Xi \\\|\xi\|<X}} \sum_{\substack{\gamma \in \Gamma \\\|\gamma\|<T}} e\left(\theta\left\langle\mathbf{v}_{0} \cdot \xi \gamma, \mathbf{w}_{0}\right\rangle\right),
$$

for certain parameters $X$ and $T$ chosen optimally in relation to $N$. One uses the full sum over the group $\Gamma$ to capture the major arcs and admissibility conditions. For the minor arcs bound, one keeps $\gamma$ fixed and uses the classical arithmetic group $\Xi$ to get sufficient cancellation to prove the desired bound (5.17). Again, we spare the reader all details.

5.4. Conclusion. Putting together the above-sketched minor arcs upper bound (5.17) with the major arcs lower bound (5.13) discussed in $\$ 5.2$, we prove the main Theorem $X$, as explained in $\$ 5.1$. We end by emphasizing again that, though the problems have nearly identical reformulations, the circle method is only a method and not an applicable theorem: while the idea of breaking the integral (5.8) into

\section{TABLE 1}

\begin{tabular}{c|c|c} 
Theorem & Tools for Major Arcs & Ingredients for Minor Arcs \\
\hline A & $\begin{array}{c}\text { infinite volume } \\
\text { hyperbolic } 3 \text {-folds, } \\
\text { automorphic forms, } \\
\text { representations, expanders }\end{array}$ & $\begin{array}{c}\text { that } \Gamma \text { contains the arithmetic } \\
\text { subgroup } \Xi \cong \Lambda(2), \\
\text { bilinear forms }\end{array}$ \\
\hline P & $\begin{array}{c}\text { infinite volume } \\
\text { hyperbolic 2-folds, } \\
\text { automorphic forms, } \\
\text { representations, expanders }\end{array}$ & $\begin{array}{c}\text { replacing } \Gamma \text { by } \mathrm{SL}_{2}(\mathbb{Z}) \\
\text { for } \delta \text { near } 1, \\
\text { bilinear forms }\end{array}$ \\
\hline Z & $\begin{array}{c}\text { thermodynamic formalism, } \\
\text { congruence transfer operators, } \\
\text { expanders }\end{array}$ & $\begin{array}{c}\text { replacing } \Gamma_{\mathcal{A}} \text { by } \mathrm{SL}_{2}(\mathbb{Z}) \\
\text { for } \delta_{\mathcal{A}} \text { near } 1, \\
\text { bilinear forms }\end{array}$
\end{tabular}


major and minor arcs is ubiquitous, the actual execution of this idea is handled by very different tools in each case; see Table 1 . Besides the circle method, the only other pervasive and critical ingredients are expanders for the major arcs and bilinear forms for the minor arcs.

\section{ACKNOWLEDGMENTS}

The author wishes to thank Andrew Granville for encouraging him to pen these notes and for his insightful and detailed input on various drafts. The author also thanks Mel Nathanson for inviting him to give a mini-course at CANT 2012, as a result of which these notes were finally assembled. The author is grateful to Peter Sarnak for introducing him to Apollonian gaskets and infinite volume spectral methods, to Hee Oh for introducing him to homogeneous dynamics, and to Dorian Goldfeld for his constant support and advice. Thanks to Elena Fuchs, Aryeh Kontorovich, Sam Payne, and especially the referee for detailed comments on an earlier draft. Most of all, the author owes a huge debt of gratitude to Jean Bourgain for his generous tutelage and collaboration.

\section{ABOut THE AUTHOR}

Alex Kontorovich is assistant professor of mathematics at Yale University. He received his Ph.D. at Columbia University in 2007 under Dorian Goldfeld and Peter Sarnak, and has held positions at Brown University, the Institute for Advanced Study, and Stony Brook University.

\section{REFERENCES}

[BF11] Jean Bourgain and Elena Fuchs, A proof of the positive density conjecture for integer Apollonian circle packings, J. Amer. Math. Soc. 24 (2011), no. 4, 945-967, DOI 10.1090/S0894-0347-2011-00707-8. MR2813334 (2012d:11072)

[BG08] Jean Bourgain and Alex Gamburd, Uniform expansion bounds for Cayley graphs of $\mathrm{SL}_{2}\left(\mathbb{F}_{p}\right)$, Ann. of Math. (2) $\mathbf{1 6 7}$ (2008), no. 2, 625-642, DOI 10.4007/annals.2008.167.625. MR2415383 (2010b:20070)

[BGS06] Jean Bourgain, Alex Gamburd, and Peter Sarnak, Sieving and expanders, C. R. Math. Acad. Sci. Paris 343 (2006), no. 3, 155-159, DOI 10.1016/j.crma.2006.05.023 (English, with English and French summaries). MR2246331 (2007b:11139)

[BGS10] Jean Bourgain, Alex Gamburd, and Peter Sarnak, Affine linear sieve, expanders, and sum-product, Invent. Math. 179 (2010), no. 3, 559-644, DOI 10.1007/s00222-009-02253. MR2587341 (2011d:11018)

[BGS11] Jean Bourgain, Alex Gamburd, and Peter Sarnak, Generalization of Selberg's $\frac{3}{16}$ theorem and affine sieve, Acta Math. 207 (2011), no. 2, 255-290, DOI 10.1007/s11511012-0070-x. MR2892611

[BGT11] Emmanuel Breuillard, Ben Green, and Terence Tao, Approximate subgroups of linear groups, Geom. Funct. Anal. 21 (2011), no. 4, 774-819, DOI 10.1007/s00039-011-0122-y. MR 2827010

[BK10] Jean Bourgain and Alex Kontorovich, On representations of integers in thin subgroups of $\mathrm{SL}_{2}(\mathbb{Z})$, Geom. Funct. Anal. 20 (2010), no. 5, 1144-1174, DOI 10.1007/s00039-0100093-4. MR2746949 (2012i:11008)

[BK11] Jean Bourgain and Alex Kontorovich, On Zaremba's conjecture, C. R. Math. Acad. Sci. Paris 349 (2011), no. 9-10, 493-495, DOI 10.1016/j.crma.2011.03.023 (English, with English and French summaries). MR 2802911 (2012e:11012)

[BK12] J. Bourgain and A. Kontorovich. On the local-global conjecture for integral Apollonian gaskets, 2012. Preprint, arXiv:1205.4416v1.

[BKS10] Jean Bourgain, Alex Kontorovich, and Peter Sarnak, Sector estimates for hyperbolic isometries, Geom. Funct. Anal. 20 (2010), no. 5, 1175-1200, DOI 10.1007/s00039-0100092-5. MR 2746950 
[BKT04] J. Bourgain, N. Katz, and T. Tao, A sum-product estimate in finite fields, and applications, Geom. Funct. Anal. 14 (2004), no. 1, 27-57, DOI 10.1007/s00039-004-0451-1. MR2053599 (2005d:11028)

[Bou08] Jean Bourgain, The sum-product theorem in $\mathbb{Z}_{q}$ with $q$ arbitrary, J. Anal. Math. 106 (2008), 1-93, DOI 10.1007/s11854-008-0044-2. MR2448982 (2009i:11010)

[Boy73] David W. Boyd, The residual set dimension of the Apollonian packing, Mathematika 20 (1973), 170-174. MR0493763 (58 \#12732)

[Boy82] David W. Boyd, The sequence of radii of the Apollonian packing, Math. Comp. 39 (1982), no. 159, 249-254, DOI 10.2307/2007636. MR658230 (83i:52013)

[BV11] Jean Bourgain and Péter P. Varjú, Expansion in $S L_{d}(\mathbf{Z} / q \mathbf{Z}), q$ arbitrary, Invent. Math. 188 (2012), no. 1, 151-173, DOI 10.1007/s00222-011-0345-4. MR2897695

[Cas78] J. W. S. Cassels, Rational quadratic forms, London Mathematical Society Monographs, vol. 13, Academic Press Inc. [Harcourt Brace Jovanovich Publishers], London, 1978. MR:522835(80m:10019)

[Cox68] H. S. M. Coxeter, The problem of Apollonius, Amer. Math. Monthly 75 (1968), 5-15. MR.0230204 (37 \#5767)

[Des01] Rene Descartes. Euvres, volume 4. Paris, 1901. C. Adams and P. Tannery, eds.

[Dol98] Dmitry Dolgopyat, On decay of correlations in Anosov flows, Ann. of Math. (2) 147 (1998), no. 2, 357-390, DOI 10.2307/121012. MR.1626749 (99g:58073)

[DRS93] W. Duke, Z. Rudnick, and P. Sarnak, Density of integer points on affine homogeneous varieties, Duke Math. J. 71 (1993), no. 1, 143-179, DOI 10.1215/S0012-7094-93-071074. MR1230289 (94k:11072)

[DSV03] Giuliana Davidoff, Peter Sarnak, and Alain Valette, Elementary number theory, group theory, and Ramanujan graphs, London Mathematical Society Student Texts, vol. 55, Cambridge University Press, Cambridge, 2003. MR1989434 (2004f:11001)

[EL07] Nicholas Eriksson and Jeffrey C. Lagarias, Apollonian circle packings: number theory. II. Spherical and hyperbolic packings, Ramanujan J. 14 (2007), no. 3, 437-469, DOI 10.1007/s11139-007-9052-6. MR2357448(2008i:11095)

[EM93] Alex Eskin and Curt McMullen, Mixing, counting, and equidistribution in Lie groups, Duke Math. J. 71 (1993), no. 1, 181-209, DOI 10.1215/S0012-7094-93-07108-6. MR:1230290 (95b:22025)

[FS11] Elena Fuchs and Katherine Sanden, Some experiments with integral Apollonian circle packings, Exp. Math. 20 (2011), no. 4, 380-399, DOI 10.1080/10586458.2011.565255. MR 2859897 (2012j:52039)

[Fuc11] Elena Fuchs, Strong approximation in the Apollonian group, J. Number Theory 131 (2011), no. 12, 2282-2302, DOI 10.1016/j.jnt.2011.05.010. MR2832824 (2012g:11123)

[Gam02] Alex Gamburd, On the spectral gap for infinite index "congruence" subgroups of $\mathrm{SL}_{2}(\mathbf{Z})$, Israel J. Math. 127 (2002), 157-200, DOI 10.1007/BF02784530. MR.1900698 (2003b:11050)

[GLM03] Ronald L. Graham, Jeffrey C. Lagarias, Colin L. Mallows, Allan R. Wilks, and Catherine H. Yan, Apollonian circle packings: number theory, J. Number Theory 100 (2003), no. 1, 1-45, DOI 10.1016/S0022-314X(03)00015-5. MR1971245 (2004d:11055)

[GLM05] Ronald L. Graham, Jeffrey C. Lagarias, Colin L. Mallows, Allan R. Wilks, and Catherine H. Yan, Apollonian circle packings: geometry and group theory. I. The Apollonian group, Discrete Comput. Geom. 34 (2005), no. 4, 547-585, DOI 10.1007/s00454-0051196-9. MR2173929 (2009a:11090a)

[GLM06a] Ronald L. Graham, Jeffrey C. Lagarias, Colin L. Mallows, Allan R. Wilks, and Catherine H. Yan, Apollonian circle packings: geometry and group theory. II. SuperApollonian group and integral packings, Discrete Comput. Geom. 35 (2006), no. 1, 1-36, DOI 10.1007/s00454-005-1195-x. MR2183489(2009a:11090b)

[GLM06b] Ronald L. Graham, Jeffrey C. Lagarias, Colin L. Mallows, Allan R. Wilks, and Catherine H. Yan, Apollonian circle packings: geometry and group theory. III. Higher dimensions, Discrete Comput. Geom. 35 (2006), no. 1, 37-72, DOI 10.1007/s00454-005-11978. MR2183490 (2009a:11090c)

[Gra95] Andrew Granville, Harald Cramér and the distribution of prime numbers, Scand. Actuar. J. 1 (1995), 12-28, DOI 10.1080/03461238.1995.10413946. Harald Cramér Symposium (Stockholm, 1993). MR1349149 (96g:01002) 
[Hel08] H. A. Helfgott, Growth and generation in $\mathrm{SL}_{2}(\mathbb{Z} / p \mathbb{Z})$, Ann. of Math. (2) 167 (2008), no. 2, 601-623, DOI 10.4007/annals.2008.167.601. MR.2415382 (2009i:20094)

[Hen89] Doug Hensley, The distribution of badly approximable numbers and continuants with bounded digits, Théorie des nombres (Quebec, PQ, 1987), de Gruyter, Berlin, 1989, pp. 371-385. MR.1024576 (91e:11078)

[Hen92] Doug Hensley, Continued fraction Cantor sets, Hausdorff dimension, and functional analysis, J. Number Theory 40 (1992), no. 3, 336-358, DOI 10.1016/0022314X(92)90006-B. MR.1154044 (93c:11058)

[Hen96] Douglas Hensley, A polynomial time algorithm for the Hausdorff dimension of continued fraction Cantor sets, J. Number Theory 58 (1996), no. 1, 9-45, DOI 10.1006/jnth.1996.0058. MR 1387719(97i:11085a)

[JP01] Oliver Jenkinson and Mark Pollicott, Computing the dimension of dynamically defined sets: $E_{2}$ and bounded continued fractions, Ergodic Theory Dynam. Systems 21 (2001), no. 5, 1429-1445, DOI 10.1017/S0143385701001687. MR.1855840 (2003m:37027)

[KO11] Alex Kontorovich and Hee Oh, Apollonian circle packings and closed horospheres on hyperbolic 3-manifolds, J. Amer. Math. Soc. 24 (2011), no. 3, 603-648, DOI 10.1090/S0894-0347-2011-00691-7. With an appendix by Oh and Nimish Shah. $\operatorname{MR} 2784325$

[KO12] Alex Kontorovich and Hee Oh, Almost prime Pythagorean triples in thin orbits, J. Reine Angew. Math. 667 (2012), 89-131. MR2929673

[Kon07] Alex V. Kontorovich, The hyperbolic lattice point count in infinite volume with applications to sieves, ProQuest LLC, Ann Arbor, MI, 2007. Thesis (Ph.D.)-Columbia University. MR2710911

[Kon09] Alex V. Kontorovich, The hyperbolic lattice point count in infinite volume with applications to sieves, Duke Math. J. 149 (2009), no. 1, 1-36, DOI 10.1215/00127094-2009035. MR2541126 (2011f:11125)

[Lal89] Steven P. Lalley, Renewal theorems in symbolic dynamics, with applications to geodesic flows, non-Euclidean tessellations and their fractal limits, Acta Math. 163 (1989), no. 1-2, 1-55, DOI 10.1007/BF02392732. MR1007619 (91c:58112)

[Lan08] E. Landau. Über die Einteilung der positiven ganzen Zahlen in vier Klassen nach der Mindestzahl der zu ihrer additiven Zusammensetzung erforderlichen Quadrate. Arch. der Math. u. Phys., 13(3):305-312, 1908.

[LMW02] Jeffrey C. Lagarias, Colin L. Mallows, and Allan R. Wilks, Beyond the Descartes circle theorem, Amer. Math. Monthly 109 (2002), no. 4, 338-361, DOI 10.2307/2695498. MR.1903421 (2003e:51030)

[LO12] M. Lee and H. Oh. Effective circle count for Apollonian packings and closed horospheres, 2012. Preprint, arXiv:1202.1067.

[LP82] Peter D. Lax and Ralph S. Phillips, The asymptotic distribution of lattice points in Euclidean and non-Euclidean spaces, J. Funct. Anal. 46 (1982), no. 3, 280-350, DOI 10.1016/0022-1236(82)90050-7. MR661875 (83j:10057)

[Lub12] Alexander Lubotzky, Expander graphs in pure and applied mathematics, Bull. Amer. Math. Soc. (N.S.) 49 (2012), no. 1, 113-162, DOI 10.1090/S0273-0979-2011-01359-3. MR2869010(2012m:05003)

[Mar54] J. M. Marstrand, Some fundamental geometrical properties of plane sets of fractional dimensions, Proc. London Math. Soc. (3) 4 (1954), 257-302. MR0063439 (16,121g)

[McM98] Curtis T. McMullen, Hausdorff dimension and conformal dynamics. III. Computation of dimension, Amer. J. Math. 120 (1998), no. 4, 691-721. MR1637951(2000d:37055)

[MVW84] C. R. Matthews, L. N. Vaserstein, and B. Weisfeiler, Congruence properties of Zariskidense subgroups. I, Proc. London Math. Soc. (3) 48 (1984), no. 3, 514-532, DOI 10.1112/plms/s3-48.3.514. MR735226 (85d:20040)

[Nau05] Frédéric Naud, Expanding maps on Cantor sets and analytic continuation of zeta functions, Ann. Sci. École Norm. Sup. (4) 38 (2005), no. 1, 116-153, DOI 10.1016/j.ansens.2004.11.002 (English, with English and French summaries). MR2136484(2006e:37033)

[Nie78] Harald Niederreiter, Quasi-Monte Carlo methods and pseudo-random numbers, Bull. Amer. Math. Soc. 84 (1978), no. 6, 957-1041, DOI 10.1090/S0002-9904-1978-14532-7. MR.508447 (80d:65016) 
[Nov55] P. S. Novikov, Ob algoritmičeskoŭ nerazrešimosti problemy toždestva slov $v$ teorii grupp, Trudy Mat. Inst. im. Steklov. no. 44, Izdat. Akad. Nauk SSSR, Moscow, 1955 (Russian). MR0075197 (17,706b)

[OEI] http://oeis.org/A195901.

[Oh10] Hee Oh, Dynamics on geometrically finite hyperbolic manifolds with applications to Apollonian circle packings and beyond, Proceedings of the International Congress of Mathematicians. Volume III, Hindustan Book Agency, New Delhi, 2010, pp. 13081331. MR2827842(2012f:37004)

[OS12] Hee Oh and Nimish Shah, The asymptotic distribution of circles in the orbits of Kleinian groups, Invent. Math. 187 (2012), no. 1, 1-35, DOI 10.1007/s00222-0110326-7. MR2874933 (2012k:37011)

[Pat76] S. J. Patterson, The limit set of a Fuchsian group, Acta Math. 136 (1976), no. 3-4, 241-273. MR0450547 (56 \#8841)

[Pli] http://en.wikipedia.org/wiki/Plimpton_322.

[PS10] L. Pyber and E. Szabo. Growth in finite simple groups of lie type of bounded rank, 2010. Preprint arXiv: 1005.1858.

[Rap12] A. Rapinchuk. On strong approximation for algebraic groups, 2012. Preprint arXiv: 1207.4425

[Sar04] Peter Sarnak, What is... an expander?, Notices Amer. Math. Soc. 51 (2004), no. 7, 762-763. MR2072849

[Sar07] P. Sarnak. Letter to J. Lagarias, 2007. http://web.math.princeton.edu/sarnak/ AppolonianPackings.pdf.

[Sar08] Peter Sarnak, Equidistribution and primes, Astérisque 322 (2008), 225-240. Géométrie différentielle, physique mathématique, mathématiques et société. II. MR2521658 (2010k:11146)

[Sar11] Peter Sarnak, Integral Apollonian packings, Amer. Math. Monthly 118 (2011), no. 4, 291-306, DOI 10.4169/amer.math.monthly.118.04.291. MR2800340 (2012e:52047)

[Sch72] Wolfgang M. Schmidt, Irregularities of distribution. VII, Acta Arith. 21 (1972), 45-50. MR 0319933 (47 \#8474)

[SG12] Alireza Salehi Golsefidy, Counting lattices in simple Lie groups: the positive characteristic case, Duke Math. J. 161 (2012), no. 3, 431-481, DOI 10.1215/00127094-1507421. MR2881228(2012k:22015)

[SGS11] A. Salehi Golsefidy and P. Sarnak. Affine sieve, 2011. Preprint.

[SGV11] A. Salehi Golsefidy and P. Varju. Expansion in perfect groups, 2011. Preprint.

[Sod36] F. Soddy. The kiss precise. Nature, 137:1021, 1936.

[Sod37] F. Soddy. The bowl of integers and the hexlet. Nature, 139:77-79, 1937.

[Sul84] Dennis Sullivan, Entropy, Hausdorff measures old and new, and limit sets of geometrically finite Kleinian groups, Acta Math. 153 (1984), no. 3-4, 259-277, DOI 10.1007/BF02392379. MR766265 (86c:58093)

[SX91] Peter Sarnak and Xiao Xi Xue, Bounds for multiplicities of automorphic representations, Duke Math. J. 64 (1991), no. 1, 207-227, DOI 10.1215/S0012-7094-91-06410-0. MR $1131400(92 \mathrm{~h}: 22026)$

[Var10] P. Varju. Expansion in $S L_{d}\left(O_{K} / I\right), I$ square-free, 2010. arXiv:1001.3664v1.

[Vin37] I. M. Vinogradov. Representation of an odd number as a sum of three primes. Dokl. Akad. Nauk SSSR, 15:291-294, 1937.

[Vin12] I. Vinogradov. Effective bisector estimate with application to Apollonian circle packings, 2012. Princeton University Thesis, arxiv:1204.5498v1.

[Zar66] S. C. Zaremba, Good lattice points, discrepancy, and numerical integration, Ann. Mat. Pura Appl. (4) 73 (1966), 293-317. MR0218018 (36 \#1107)

[Zar72] S. K. Zaremba, La méthode des "bons treillis" pour le calcul des intégrales multiples, Applications of number theory to numerical analysis (Proc. Sympos., Univ. Montreal, Montreal, Que., 1971), Academic Press, New York, 1972, pp. 39-119 (French, with English summary). MR0343530 (49 \#8271)

Department of Mathematics, Yale University, New Haven, Connecticut

E-mail address: alex.kontorovich@yale.edu 